\title{
Epoxy/imidazole functionalized silica epoxy nanocomposites: Mechanical and fracture behaviour
}

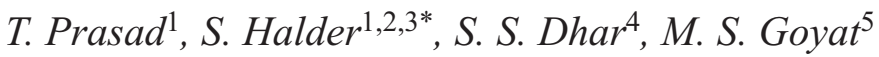 \\ ${ }^{1}$ Department of Mechanical Engineering, National Institute of Technology Silchar, Silchar, 788010 Assam, India \\ ${ }^{2}$ Alabama Transportation Institute, The University of Alabama, AL 35487 Tuscaloosa, USA \\ ${ }^{3}$ Aerospace Engineering and Mechanics, Center for Advanced Vehicle Technologies, AL 35487 Tuscaloosa, USA \\ ${ }^{4}$ Department of Chemistry, National Institute of Technology Silchar, Silchar, 788010 Assam, India \\ ${ }^{5}$ Department of Applied Science, School of Engineering, University of Petroleum and Energy Studies, 28007 Dehrdaun, \\ India
}

Received 7 May 2020; accepted in revised form 29 July 2020

\begin{abstract}
In this work, the mechanical and fracture performance of epoxy nanocomposites consisting of epoxide and imidazole functionalized silica nanoparticles has been studied. The post-synthesis grafting method was utilized to functionalized $\mathrm{SiO}_{2}$ nanoparticles with GPTMS (GGS) and used them as reinforcement (0-2 wt \%) in epoxy resin. The cure behavior of nanocomposites demonstrated that the composite has excellent cure capability at $0.5 \mathrm{wt} \%$ of $G G S$. The composite containing $0.5 \mathrm{wt} \%$ of $G G S$ exhibited significant improvement in tensile strength $(\sim 65 \%)$ and modulus of toughness $(\sim 272 \%)$, respectively. Additionally, the flexural strength, flexural modulus, and work of flexural were enhanced by $\sim 48, \sim 50$, and $\sim 48 \%$, respectively. Interestingly, the $G G S$ showed its tremendous potential to improve the fracture toughness $\left(K_{1 \mathrm{C}}\right)$ and the fracture energy $\left(G_{1 \mathrm{C}}\right)$ of the nanocomposite by $\sim 97$ and $\sim 292 \%$, which is also evident by the study of cure behavior. The fractography analysis endorsed the enhancement of material properties due to the use of $G G S$ in the epoxy matrix. Failure investigation examined under FESEM elucidated forced the crack to move around the poles of the nanoparticles due to better interfacial adhesion. Hence, GGS nanoparticle has the potential to use as an excellent cost-effective reinforcement for the epoxy matrix to mitigate the brittle failure in epoxy composites.
\end{abstract}

Keywords: nanocomposites, interface, cure index, tensile strength, fracture toughness

\section{Introduction}

Over the past few decades, researchers and scientists devoted colossal interest to modify epoxy polymer network with nanoparticle for the enriching of their utilization in the various advanced sector of industries such as defense, wind energy, aerospace, automotive, and locomotive, etc. [1-4]. The primary aim of reinforcing nanofillers is to overcome the intrinsic drawback of the epoxy resin system in terms of their low toughness and stiffness or rigidity. These properties are mostly dependent on the crosslinking density of the epoxy system. A higher crosslinking density of the epoxy system increases the chances of brittle fracture. Numerous works demonstrated a marginal effect of de-agglomerated nanofillers on tailoring the crosslinking of the epoxy system and ends up with enhanced catastrophic failure and minimal enhancement of fracture toughness [5]. In the fracture surfaces of nanofiller reinforced epoxy composite, smaller fracture pieces are evident in the cleavage zones endorsing hindrance offered by nanoparticles towards crosslinking [6]. Thus, the modulation of the crosslinking density of epoxy systems by adding bare 
nanofillers even after de-aggregation and proper dispersion in the matrix is highly questionable.

Low-cost inorganic nanoparticles such as silica [7], nano clay [8], zinc oxide [9, 10], and zirconia [11] is an excellent choice for plentiful advanced composite structure manufacturing. For example, epoxy/ silica composites have been widely used as cryogenic gas storage tanks in the aerospace industries [12]. The silica nanoparticles have been extensively used as a reinforcement for epoxy polymer not only due to their high surface areas, high tensile strength, good in thermal stability, and low specific density but also because of ease of bulk synthesis with high purity and narrow particle size distribution [13]. However, to date, few studies can be found that elaborate on the change in the crosslinking behavior of the epoxy system by using surface-functionalized silica nanofiller and its effect on mitigating catastrophic failure [14]. Silane modified silica in the recent past has been introduced in many applications such as coatings, wind turbine blades, structural adhesives, and car tires, etc. [15-17]. It is most likely that the overall performance of composites mainly depends on the surface chemistry of modified silica nanoparticles and wettability towards dispersion in the epoxy matrix.

In comparison to other inorganic reinforcements, silica nanoparticles are very prone to aggregation, especially in the most viscous epoxy system owing to their hygroscopic nature resulting in poor adhesion between the fillers and the matrix. Due to the high viscosity of resin mixture, the use of a low boiling inert solvent may improve the homogeneous distribution of silica particles in epoxy resin. Nevertheless, there is a chance that the solvent trace entrapped within the resin mixture even after curing may lead to deteriorating the mechanical and fracture properties of the composites [18]. Modification with coupling agents can be another promising approach to avoid agglomeration and probably improve the adhesion between fillers and matrix [19]. Since the silane coupling agent acts as a chemical bridge between hydrophilic $\mathrm{SiO}_{2}$ and epoxy matrix. The enhanced interfacial adhesion by covalently functionalized silica nanoparticles with epoxy networks can overcome such difficulties. The commonly used coupling agents in an amine cured epoxy system are introduced either as epoxy or amino silane-based coupling agents. It was reported that the epoxy silane is more effective in dispersing the silica nanoparticles as compared to the amino silane due to the absence of hydrogen bonding between the particles [20].

Recently, few efforts address the effect of amino silane functionalized $\mathrm{SiO}_{2}$ on the mechanical and fracture properties of the epoxy system. For instance, Kim et al. [21] reported the influence of 3-aminopropyltriethoxy silane functionalized $\mathrm{Al}_{2} \mathrm{O}_{3}$ nanoparticles on the mechanical property of epoxy. They demonstrated the tensile strength and the tensile modulus enhancement by $\sim 12$ and $\sim 9 \%$, respectively, as compared to neat epoxy. Similarly, Han et al. [22] reported fracture toughness improvement of the aminefunctionalized silica by $\sim 25 \%\left(1.15 \mathrm{MPa} \cdot \mathrm{m}^{0.5}\right)$ whereas, epoxy functionalized silica improves the fracture toughness by $\sim 36 \%\left(1.25 \mathrm{MPa} \cdot \mathrm{m}^{0.5}\right)$ with respect to neat $\left(0.92 \mathrm{MPa} \cdot \mathrm{m}^{0.5}\right)$. Further, Zhang et al. [23] reported the influence of supported ionic liquid silica as a curing agent on the mechanical properties of epoxy composites. They demonstrated maximum enhancement of $\sim 13 \%$ (52 MPa), $20 \%(84 \mathrm{MPa})$, $\sim 9 \%(2.32 \mathrm{GPa})$, and $\sim 17 \%\left(19.2 \mathrm{~kJ} \cdot \mathrm{m}^{-2}\right)$ in tensile strength, flexural strength, flexural modulus, impact strength respectively as compare to neat epoxy. Recently, Wang et al. [12] reported the effect of different silane coupling agents on the cryogenic properties of silica-epoxy nanocomposites. They demonstrated that composite modified with epoxy silane had a superior performance than that of composite modified with an amino silane. However, hardly any published reports reflect the analysis depicting the effect of epoxy functionalized silica on curing, mechanical and fracture properties of epoxy nanocomposites. This gap gives us the essence of scrutinizing epoxy silane grafted silica nanoparticle to use as reinforcement in the epoxy system.

In the present study, we functionalized $\mathrm{SiO}_{2}$ nanoparticles with epoxy silane organic moieties through the post-synthesis grafting method. They were reinforced in the epoxy matrix to enhance their interfacial interaction between the filler and the matrix at low silica contents. The effect of epoxy silane grafted silica on the curing, mechanical, and fracture behavior of epoxy composites is determined. Additionally, we also demonstrate fracture mechanisms to endorse the crosslinking modulation capability of the epoxy matrix comprising epoxy silane grafted silica. 


\section{Experimental details}

\subsection{Materials}

Tetraethyl-orthosilicate (TEOS, purity 98\%) was used as a precursor/reactant and ammonia solution $\left(25 \% \mathrm{NH}_{3}\right.$ in water) as a reagent/catalyst obtained from Merck Specialties Private Limited, Mumbai, India. The solvent, absolute ethanol $\left(\mathrm{C}_{2} \mathrm{H}_{5} \mathrm{OH}\right.$, purity 99.9\%) purchased from Changshu Hongsheng Fine Chemical Ltd., Jiangsu, China. The surface modifier (3-Glycidyloxypropyl) trimethoxysilane (GPTMS, purity $\geq 98 \%$ ) used as a coupling agent for the functionalization of silica nanoparticles, purchased from Alfa Aesar India Pvt. Ltd. Hyderabad, India. Another surface modifier, 1-methyl imidazole (purity $\geq 97 \%$ ) was purchase from Huntsman India Pvt. Ltd., India. The Diglycidylether of bisphenol-A (DGEBA) based epoxy resin (Lapox L12) with density $1.20 \mathrm{~g} \cdot \mathrm{cm}^{-3}$ and Triethylenetetramine, (TETA, K6) hardener with density $0.95 \mathrm{~g} \cdot \mathrm{cm}^{-3}$ was supplied by Atul ltd., Gujarat, India.

\subsection{Synthesis and functionalization of silica nanoparticles}

The silica nanoparticles were synthesized using modified sol-gel method [24]. In this process, $8 \mathrm{ml}$ $(1.0 \mathrm{M})$ of TEOS precursor was mixed with $60 \mathrm{ml}$ of ethanol and $2 \mathrm{ml}(3 \mathrm{M})$ of deionized water and added to the capacity of $500 \mathrm{ml}$ beaker. After the preparation of the homogeneous solution followed by vigorous stirring, $2.8 \mathrm{ml}(2.0 \mathrm{M})$ of $\mathrm{NH}_{4} \mathrm{OH}$ catalysis is added gently under constant stirring. The reaction was continued for 12 hours without supplying any external source of heat. The white milky gel in the slurry form is precipitated at the end of the reaction. Then the powder was separated by centrifugation and repeatedly washed with ethanol, followed by washing with distilled water. In the end, the powder was kept in a vacuum oven to dry at $100^{\circ} \mathrm{C}$ for 12 hours.

Further, the functionalization of silica with GPTMS was carried out using the post-synthesis grafting method [25]. In a typical procedure, $1 \mathrm{~g}$ silica $(A S S)$ was added in $100 \mathrm{ml}$ toluene, and the suspension was sonicated using bath type sonication for $30 \mathrm{~min}$. Subsequently, $0.71 \mathrm{~g}(6 \mathrm{mM})$ of GPTMS was added into a $250 \mathrm{ml}$ single neck round bottom flask using micropipette with stirring at $90^{\circ} \mathrm{C}$ for $12 \mathrm{~h}$ under reflux condition. The slurry was centrifuged and washed repeatedly with ethanol and distilled water. At last, the powder was kept in a vacuum oven to dry at $90^{\circ} \mathrm{C}$ for 24 hours, and the powder is coded as $G G S$. Similarly, the functionalization of silica with imidazole was carried out using the post-synthesis grafting method [26], as illustrated in Figure 1.

In a typical procedure, $1.00 \mathrm{~g}$ silica was added in $100 \mathrm{ml}$ toluene, and the suspension was sonicated using bath type sonication for $30 \mathrm{~min}$. Consecutively,

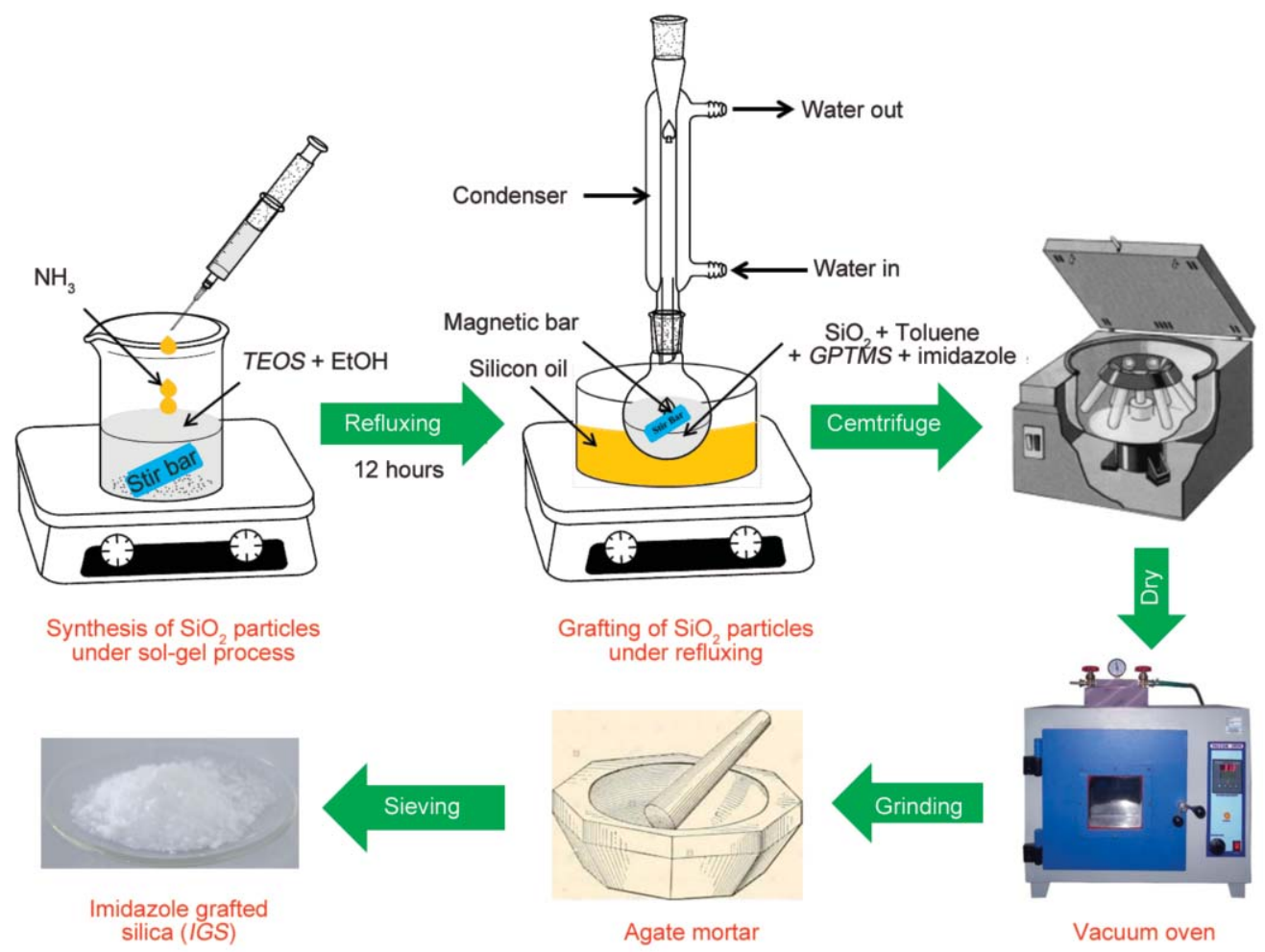

Figure 1. Schematic illustration of functionalization of post-synthesized silica with imidazole. 


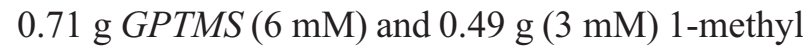
imidazole were added into a $250 \mathrm{ml}$ round bottom flask using micro-pipette with stirring at $90^{\circ} \mathrm{C}$ for $12 \mathrm{~h}$ under reflux condition in a nitrogen atmosphere. The powder was separated by centrifugation and repeatedly washed with ethanol and double-distilled deionized water. Finally, the powder was dried in a vacuum oven at $90^{\circ} \mathrm{C}$ for 24 hours, and the powder is coded as $I G S$.

\subsection{Preparation of epoxy nanocomposites}

The silica/epoxy composites were prepared by reinforcing varying contents $(0-2 \mathrm{wt} \%)$ of as-synthesized silica (ASS), GPTMS grafted silica (GGS), and imidazole grafted silica $(I G S)$ into epoxy resin and thoroughly mixed using overhead stirrer at $1400 \mathrm{rpm}$ for 10 minutes. The dispersion of each silica variants $(0.5 \mathrm{wt} \%)$ into the epoxy resin was performed by ultrasonic probe sonicator (Q-Sonica, Newtown, CT, USA) with a maximum output power of $700 \mathrm{~W}$ (Frequency: $20 \mathrm{kHz}$, amplitude: $70 \%$ ) under dual-mode mixing for another 30 minutes [27]. The temperature of the nano resin mixture was monitored continuously and maintained at room temperature by thermocouple using circulating cold water around the disposal plastic glass containing resin mixture via water bath chiller to avoid degradation. Then, stoichiometric amount 12:1 ratio of hardener K6 to epoxy resin was added to the above mix and continued mixing for another 5 minutes under overhead stirrer to obtain a homogeneous mixture followed by degassing using vacuum designator connected with a vacuum pump ( $\sim 10^{-3}$ Torr) for 40 minutes to remove the entrapped air. Then, the nano resin mixture was poured into a rubber mold cavity and kept in a vacuum oven at $80^{\circ} \mathrm{C}$ for 12 hours for curing. Similarly, other variant based nanocomposites were also prepared and designated as NE, ASEC-0.5, ASEC-1, ASEC-2, GSEC-0.5, GSEC-1, GSEC-2, ISEC-0.5, ISEC-1 and $I S E C-2$ for each composites samples such as neat epoxy, ASS embedded epoxy resin at $0.5,1$ and $2 \mathrm{wt} \%, G G S$ embedded epoxy resin at $0.5,1$, and $2 \mathrm{wt} \%$, and $I G S$ embedded epoxy resin at $0.5,1$, and $2 \mathrm{wt} \%$, respectively.

\subsection{Method of characterizations}

The particle size variation in $A S S / G G S / I G S$ after surface functionalization of silica nanoparticles was investigated using Transmission Electron Microscopy (TEM), JEM 2100, JEOL (Japan) with an operating voltage of $200 \mathrm{kV}$. The samples were prepared by sonication (30 seconds) of each nanoparticle $(0.05 \mathrm{mg} / \mathrm{ml})$ in absolute ethanol. Then, one drop of the suspension was put on a gold-coated copper grid followed by evaporating the solvent. Further, the Fourier Transform Infrared spectroscopy (FTIR) analysis was performed on a Bruker Vertex 80 spectrometer, Germany, recorder to study the surface functionalization of the $A S S / G G S / I G S$ nanoparticles in the wavenumber range of 4000 to $400 \mathrm{~cm}^{-1}$. The samples were prepared by bound the powder with $\mathrm{KBr}$ in the pellets form using pallet press. Thermogravimetric analysis (TGA) was performed on a simultaneous thermal analyzer (NETZSCH STA 449 F3 Jupiter, Germany) using $10 \mathrm{mg}$ of powder samples heated in a temperature range of 30 to $800^{\circ} \mathrm{C}$ at a heating rate of $10^{\circ} \mathrm{C} / \mathrm{min}$ under $\mathrm{N}_{2}$ atmosphere with gas purging of $30 \mathrm{ml} / \mathrm{min}$. Spectroscopy analysis of all powder samples was performed on an X-ray photoelectron spectrum (XPS, AXIS Supra, UK) with an X-ray source of $600 \mathrm{Watt}(1486.6 \mathrm{eV})$. Cure characterization of neat epoxy and epoxy nanocomposites were studied on a simultaneous thermal analyzer Differential Scanning Calorimeter (DSC), NETZSCH STA 449 F3 Jupiter (Germany). The mixture of the neat epoxy system and a mixture of nano-resin were weighted of about $15 \mathrm{mg}$ and placed in an aluminum DSC sample holder. Then, the sample was non-isothermally heated at standard heating regimes of $10{ }^{\circ} \mathrm{C} / \mathrm{min}$ in the temperature range of $40-300^{\circ} \mathrm{C}$ under nitrogen with a flow rate of $30 \mathrm{ml} / \mathrm{min}$. The mechanical test (tension test without extensometer) was performed on a computerized universal tensile machine (INSTRON, Model 5969 (USA)) at a crosshead speed of $1 \mathrm{~mm} / \mathrm{min}$. The tensile samples were prepared by following ASTM D638 (type-V) followed by grinding and polishing. The flexural and SENB samples were prepared according to ASTM D790 and ASTM D5045 standard, respectively, followed by grinding and polishing. The SENB sample was prepared with a dimension of $6 \mathrm{~mm} \times 12 \mathrm{~mm} \times$ $60 \mathrm{~mm}$ and a span of $48 \mathrm{~mm}$. Further, the notched is produced through mini hexa-blade exactly at the middle whereas, the pre-crack is generated using tape with a razor blade. However, the mechanical properties such as tensile, flexural, and fracture performance were quantitatively measured using the following mathematical relationship. Such as in tensile property, the modulus of toughness $\left(U_{\mathrm{T}}\right)$ is measured [28] in $\mathrm{MJ} / \mathrm{m}^{3}$ and can be calculated using Equation (1): 
$U_{\mathrm{T}}=\int_{0}^{\varepsilon_{\mathrm{f}}} \sigma \mathrm{d} \varepsilon \cdot 10^{6}$

where $\sigma$ is the ultimate tensile strength, $\varepsilon_{\mathrm{f}}$ is the strain at fracture and $\varepsilon$ is the tensile strain. The flexural strength $\left(\sigma_{\mathrm{f}}[\mathrm{MPa}]\right)$, is measured [29] by Equation (2):

$\sigma_{\mathrm{f}}=\frac{3 P L}{2 b d^{2}}$

where $P$ is the flexural load at a given point, $L$ is the length of support span [mm], $b$ is the width of the specimen [mm], and $d$ is the height of specimen [mm]. The flexural strain $\left(\varepsilon_{\mathrm{f}}\right)$ is determined using Equation (3):

$\varepsilon_{\mathrm{f}}=\frac{p \delta t}{L^{2}}$

where $\delta$ is the maximum/central deflection [mm] of the beam, the flexural modulus ( $\left.E_{\mathrm{f}}[\mathrm{GPa}]\right)$, can be calculated using Equation (4):

$E_{\mathrm{f}}=\frac{m L^{3}}{4 b d^{3}}$

where $L, b, d$ are the same as for the above equation, $m$ is the slope of the modulus line $[\mathrm{N} / \mathrm{mm}]$. The work of flexural $\left(\gamma\left[\mathrm{MJ} / \mathrm{m}^{2}\right]\right)$, can be calculated by Equation (5):

$$
W O F(\gamma)=\frac{U}{b d}=\frac{P \delta}{A} \cdot 10^{-6}
$$
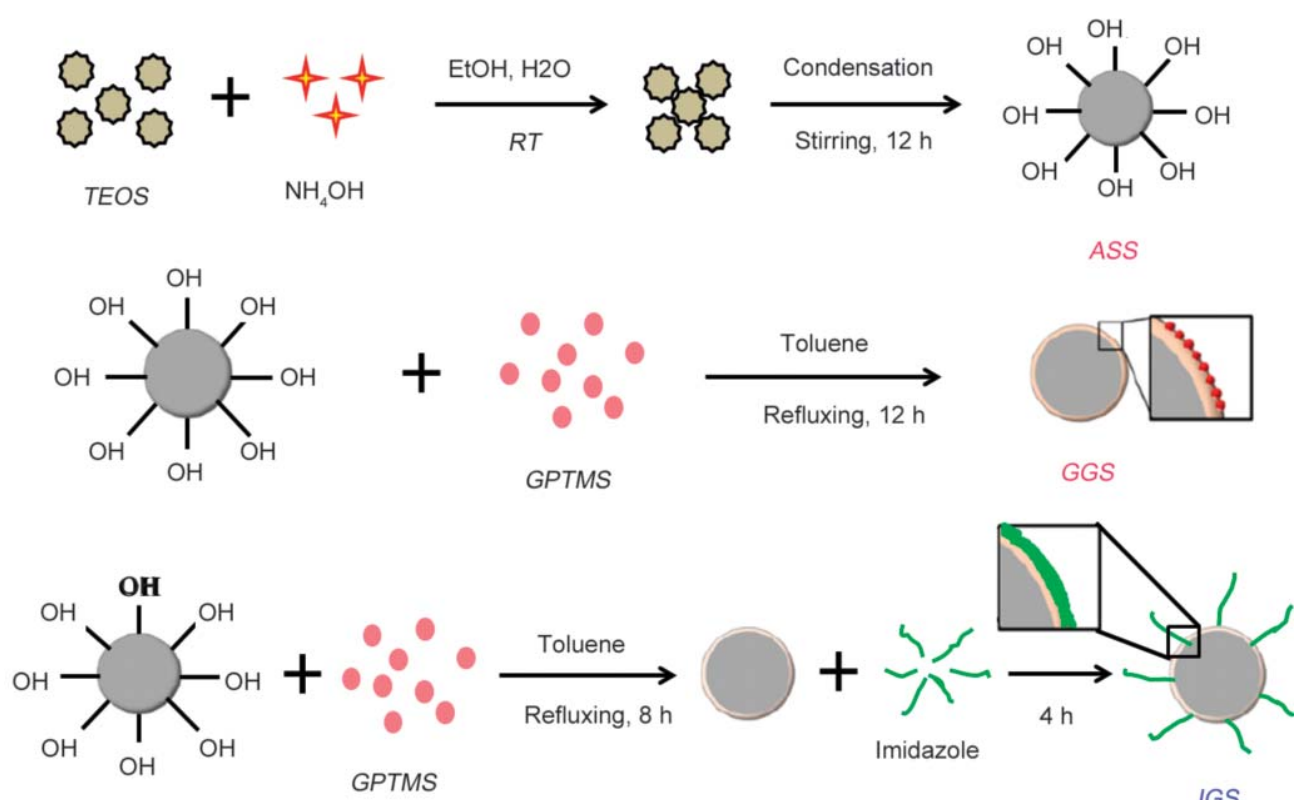

IGS

Figure 2. Schematic representation for synthesis and functionalization of silica nanoparticles. 
water with pre-determined quantities of $\mathrm{C}_{2} \mathrm{H}_{5} \mathrm{OH}$ solvent for the synthesis of silica.

During the process, TEOS was first hydrolyzed to produce the monomer of silanol groups $(-\mathrm{Si}-\mathrm{OH})$. Then, the condensation among silanol groups occurred after additions of the $\mathrm{NH}_{3}$ catalyst to create siloxane clusters ( $\mathrm{Si}-\mathrm{O}-\mathrm{Si}$ bonds) under controlled nucleation and growth of silica nuclei followed by growth termination. The growth mechanism of silica nanoparticles follows the 'monomer addition model' [30] under high $\mathrm{NH}_{3}$ concentration $(>0.90 \mathrm{M})$, as schemed in Figure 3. This model involves the faster rate of hydrolysis that led to the high conversion of silanol groups into the ethoxyl groups. This is attributed to the increase in $\mathrm{Si}^{+}$atoms that reduces the steric intermolecular interaction of ethoxyl groups and hence favored more nucleophilic attack for the formation of silanol groups. The modification of silica nanoparticles with GPTMS is described in Figure 3b. The epoxy groups of silane are catalyzed in aqueous solutions to form silanol groups. The siloxane linkage between the silica surface and the silane molecules is then produced through a polycondensation reaction. The modification of silica nanoparticles with imidazole is described in Figure 3c. The epoxy groups of silane are catalyzed to form silanol groups.
The hydroxyl group of GPTMS is later bonded onto the silica surface.

The siloxane linkage between the silica surface and the silane molecules was then produced with the addition of a ring-opening catalyst under high temperature refluxing, the imidazole attached to the silica surface through the GPTMS coupling linkage with the help of covalent bonding between the carbon and the pyridine nitrogen of imidazole. Thus the bonding of the imidazole group to GPTMS grafted silica continues under the nucleophilic attack of pyridine type of nitrogen [31]. Based on this, the final chemical structure of $I G S$ is proposed.

\subsection{Microscopy investigation of nanoparticles}

The TEM analysis of the nanoparticles is done to find out the particle size variation in silica nanoparticles before and after functionalization. The microscopy investigation shows a larger particle size of $G G S(24 \mathrm{~nm})$ and $I G S(69 \mathrm{~nm})$ as compared to ASS nanoparticles after functionalization. Variation in the size of $A S S, G G S$, and $I G S$ are found after grafting of nanoparticle with organic moieties, as shown in Figure 4.

TEM micrograph shows the particle size of $A S S$ as $\sim 345 \mathrm{~nm}$ (Figure 4a), $369 \mathrm{~nm}$ for GGS (Figure 4b),
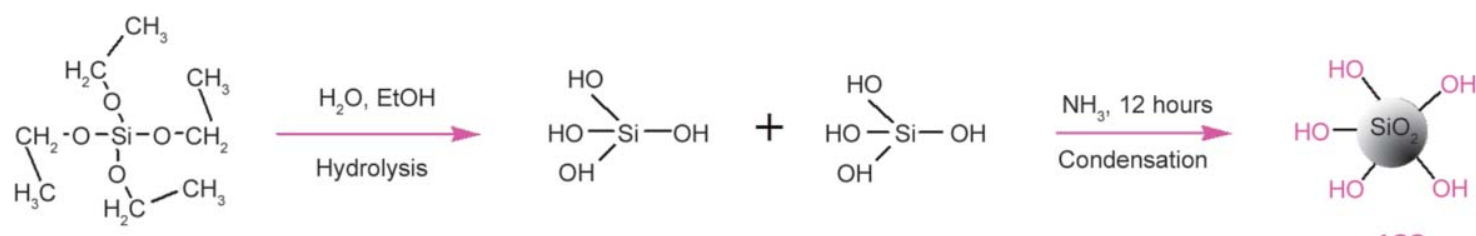

a)

TEOS
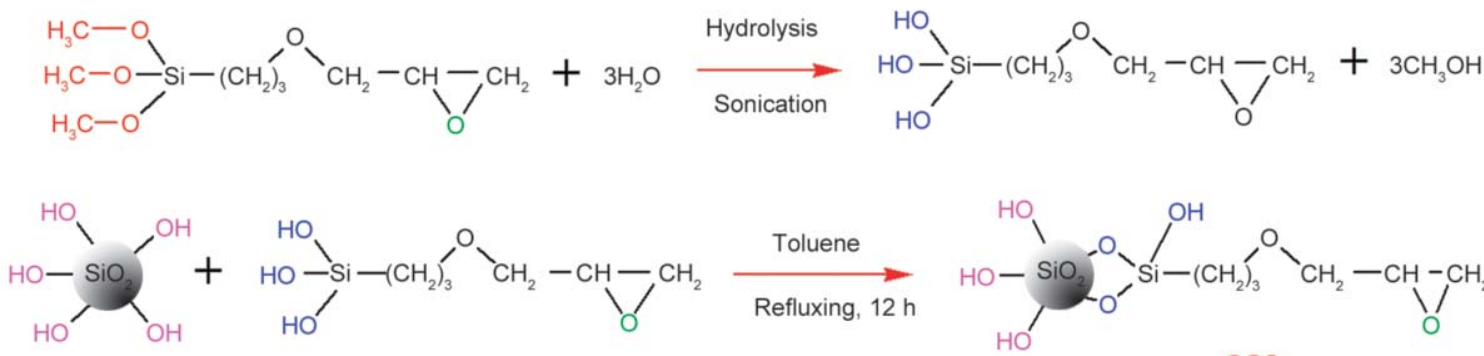

b)
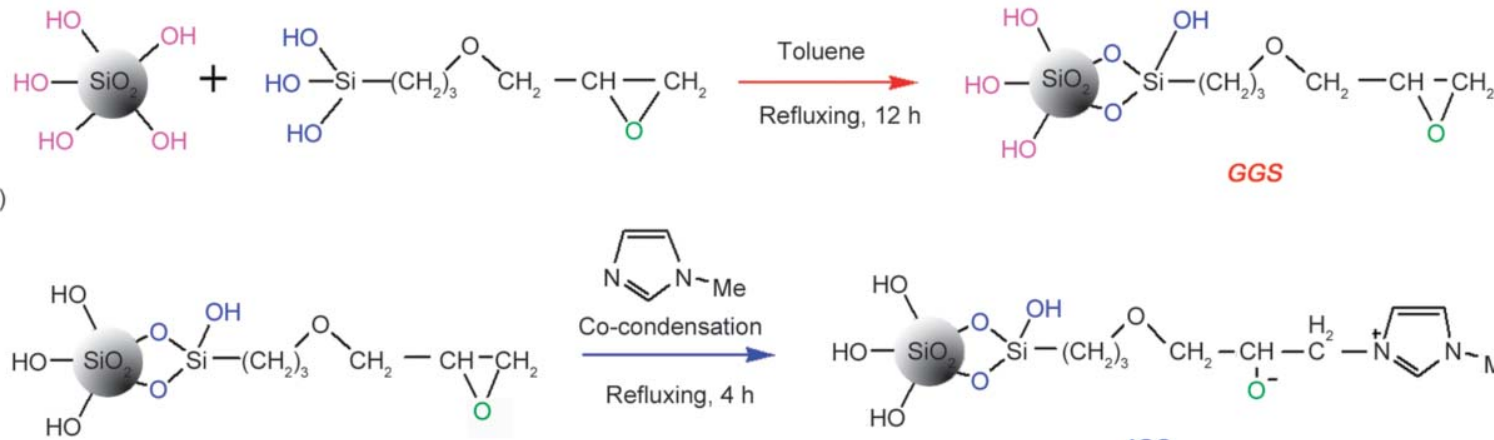

c)

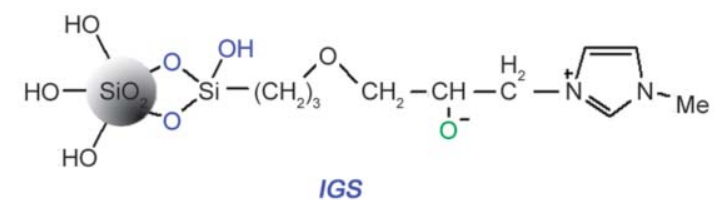

Figure 3. A plausible reaction mechanism for synthesis and functionalization of $\mathrm{SiO}_{2}$ nanoparticles (a) $A S S$ (b) GGS and (c) IGS nanoparticles. 


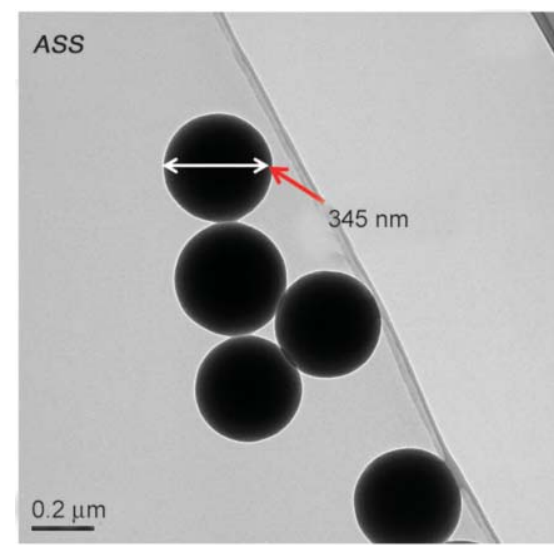

a)

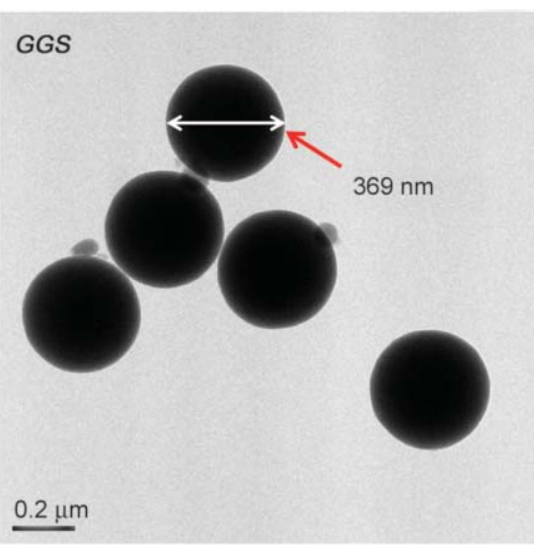

b)

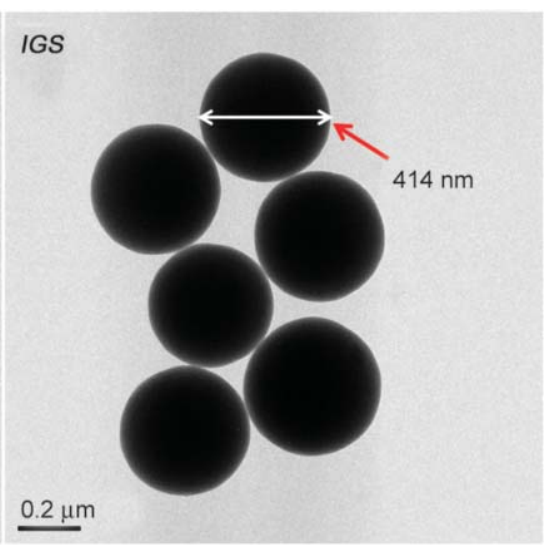

c)

Figure 4. HR-TEM micrographs of bare and functionalized silica nanoparticle (a) $A S S$ (b) $G G S$ and (c) $I G S$.

and $414 \mathrm{~nm}$ for $I G S$ (Figure 4c), respectively. The increment in the size of GGS compared to ASS is possibly attributed to the inter-linkage of silane molecules to the hydroxyl group of silica nanoparticles. Further increase in the size of IGS is predicted from controlling the degree of condensation between hydrolyzed GPTMS grafted silica and imidazole. Since imidazole is nucleophilic, therefore pyridine nitrogen from imidazole increases the catalysis resulting in the formation of a covalent bond between imidazole and epoxide group of GPTMS. This can be ascribed to the disordered self-seeding growth that caused the variation in the size of the particle. According to the classical thermodynamics approach, the surface energy is the main dominating factors that deal with variation in particle size after surface functionalization. Generally, surface energy increases with increasing particle size after functionalization, which leads to increases the binding energy between the atoms [32]. Furthermore, this compensating effect may be expected to the formation of an electric double layer at the surface.

\subsection{Investigation of functional group attachment on $\mathrm{SiO}_{2}$ nanoparticles}

The major functional group for each nanoparticle, namely $A S S, G G S$, and $I G S$, is firstly identified using FTIR spectral analysis, as reported in the earlier published work [33]. The summery on $I R$ peak details with and without functionalized silica has been given in Table 1. In brief, the peak at $\sim 805$ and $\sim 1098 \mathrm{~cm}^{-1}$ is attributed to the asymmetric and symmetric stretching vibration of $\mathrm{Si}-\mathrm{O}-\mathrm{Si}$ bond. However, the characteristic peak $\sim 940 \mathrm{~cm}^{-1}$ is assigned to the asymmetric vibration of $\mathrm{Si}-\mathrm{OH}$. This confirms the formation of silica nanoparticles. For $G G S$ and $I G S$, a new

Table 1. FTIR peak characteristics of $A S S, G G S$ and $I G S$ nanoparticles.

\begin{tabular}{|c|c|c|c|}
\hline Sample code & $\begin{array}{c}\text { Wavenumber } \\
{\left[\mathrm{cm}^{-1}\right]}\end{array}$ & Functional groups & Description \\
\hline \multirow{8}{*}{$A S S$} & 3656 & $\mathrm{Si}-\mathrm{OH}$ & Stretching vibrations of Si-OH groups \\
\hline & 3438 & $\mathrm{Si}-\mathrm{OH}$ & Stretching vibration of $\mathrm{H}_{2} \mathrm{O}$ molecules \\
\hline & 1630 & $\mathrm{Si}-\mathrm{OH}$ & Bending vibration of water molecular \\
\hline & 1098 & $\mathrm{Si}-\mathrm{O}-\mathrm{Si}$ & Symmetric stretching \\
\hline & 805 & $\mathrm{Si}-\mathrm{O}-\mathrm{Si}$ & Asymmetric stretching \\
\hline & 940 & $\mathrm{Si}-\mathrm{OH}$ & Asymmetric vibration of $\mathrm{Si}-\mathrm{OH}$ \\
\hline & 567 & $\mathrm{Si}-\mathrm{O}-\mathrm{Si}$ & Asymmetric stretching \\
\hline & 460 & $\mathrm{Si}-\mathrm{O}-\mathrm{Si}$ & Out of plane bending (racking) \\
\hline \multirow{5}{*}{$G G S$} & 2930 & $-\mathrm{CH}_{2}$ & Symmetric stretching \\
\hline & 2873 & $-\mathrm{CH}_{2}$ & Asymmetric Stretching \\
\hline & 1630 & $\mathrm{Si}-\mathrm{OH}$ & Bending vibration of $\mathrm{H}_{2} \mathrm{O}$ molecules. \\
\hline & 1222 & $\mathrm{C}-\mathrm{OH}$ & Epoxy ring breathing \\
\hline & 940 & $\mathrm{C}-\mathrm{O}-\mathrm{C}$ & Asymmetric bending of epoxide groups \\
\hline \multirow{2}{*}{$I G S$} & 1214 & $\mathrm{C}-\mathrm{N}$ & Asymmetric stretching \\
\hline & 1270 & $\mathrm{C}-\mathrm{H}$ & Asymmetric stretching \\
\hline
\end{tabular}


sharp peak at $\sim 2873$ and $\sim 2930 \mathrm{~cm}^{-1}$ is observed, which are associated with the $\left[-\left(\mathrm{CH}_{2}\right)_{\mathrm{n}}\right]$ groups of GPTMS corresponding to the symmetric and asymmetric stretching mode of $\mathrm{C}-\mathrm{H}$ band vibration.

For $G G S$, the peaks at $\sim 1222$ and $\sim 940 \mathrm{~cm}^{-1}$ represent corresponding to the epoxide group or breathing and asymmetric bending of the epoxy ring $(\mathrm{C}-\mathrm{O}-\mathrm{C})$ respectively. For $I G S$, the further broadening of a peak at $\sim 1098 \mathrm{~cm}^{-1}$ indicates the faster condensation of silane in the presence of imidazole. Furthermore, the new absorption peak at $\sim 1222$ and $\sim 1270 \mathrm{~cm}^{-1}$ for $I G S$ is ascribed to the asymmetric stretching mode of the $\mathrm{C}-\mathrm{N}$ bond and asymmetric stretching mode of the $\mathrm{C}-\mathrm{H}$ bond from 1-methyl imidazole, respectively. The presence of $\mathrm{C}-\mathrm{N}$ and $\mathrm{C}-\mathrm{H}$ bands confirms the attachment of 1-methyl imidazole on the surface of silica.

Similarly, the thermal degradation behavior of ASS, $G G S$, and $I G S$ are analyzed by TGA analysis, and the procedure adopted is very similar in earlier reported published work in detail [33]. In brief, the thermogram shows three-step weight-loss behavior for $G F S$ and $I F S$, whereas single step weight loss for $A S S$ is found. The initial weight loss for ASS is attributed to the volatilities of hydroxyl groups of silica, moisture, and water molecules. However, in the case of $G G S$ and $I G S$, weight loss under the first stage associated with the removal of physically absorbed moisture and water from the surfaces of $G G S$ and $I G S$. Weight loss from the second stage is determined as $\sim 11$ and $\sim 12 \%$ in the range of $191-445^{\circ} \mathrm{C}$ for $G G S$ and $176-385^{\circ} \mathrm{C}$ for $I G S$, respectively. This loss is attributed to the decomposition of hydrocarbon from the organic moieties of GPTMS and imidazole. Lastly, the third stage weight loss of $\sim 10$ and $\sim 12 \%$ was observed in the temperature range of $442-$ $800^{\circ} \mathrm{C}$ for $G G S$ and $429-800^{\circ} \mathrm{C}$ for $I G S$, respectively. This loss is attributed to the loss of carbon, nitrogen, and oxygen from the surfaces of GGS and IGS. A noticeably increased weight loss of $G G S$ and $I G S$ than that of ASS indicates the presence of organic functional groups on the surface of $A S S$. The TGA data is further utilized to determine the grafting yield of the epoxide/imidazole group on $\mathrm{SiO}_{2}$ nanoparticles using Equation (9) [34]:

$$
\begin{aligned}
& \text { Grafting density }\left[\frac{\mathrm{mmol}}{\mathrm{g}}\right]= \\
& \quad=\frac{W_{300}-W_{800}}{100-\left(W_{300}-W_{800}\right)} \cdot \frac{100}{M}
\end{aligned}
$$

where $W_{300}-W_{800}$ is the weight loss [wt $\%$ ] of $\mathrm{SiO}_{2}$ variants within the temperature range of $300-800^{\circ} \mathrm{C}$ and $M[\mathrm{~g} / \mathrm{mol}]$ is the molecular weight. The grafting density of GPTMS and Imidazole moiety on $G G S$ and $I G S$ nanoparticle was estimated within the temperature range of 300 to $800^{\circ} \mathrm{C}$ and is listed in Table 2. The molecular weight taken is $236.34 \mathrm{~g} / \mathrm{mol}$ for GPTMS and $82 \mathrm{~g} / \mathrm{mol}$ for imidazole moiety. Using Equation (9), the measured grafted density of GPTMS and Imidazole is 0.80 , and $2.67 \mathrm{mmol} / \mathrm{g}$ corresponds to 190.47 and $219.51 \mathrm{mg}$ on $1 \mathrm{~g}$ of bare $\mathrm{SiO}_{2}$ nanoparticles. Therefore, the grafting yield of 9 and $2 \mathrm{wt} \%$ for GPTMS and Imidazole, respective$1 \mathrm{y}$, is achieved by considering 710 and $490 \mathrm{mg}$ as the initial concentration of GPTMS and imidazole.

Further confirmation on the grafting of organic moieties, such as GPTMS and imidazole on silica, is derived from the dispersion study in non-polar solvents (toluene). The photographs of $A S S, G G S$, and IGS dispersed after 7 hours is depicted in Figure 5. The dispersions containing GPTMS and imidazole ligands grafted silica shows transparent, while $A S S$ dispersion remained cloudy and did not sediment during the 7 days period. This was expected, as the presence of silanol groups on the silica surface makes it hydrophilic. The dispersion behavior of $G G S$ and IGS sample was rather different after 7 hours, which is an indication of changing the surface hydrophilicity of the particles. In the colloidal region, the stability of particles in a colloidal dispersion depends on the balance of attractive and repulsive forces between two neighboring particles [35]. From Figure 5, the sedimentation of $G G S$ and $I G S$ samples shows that the attractive forces were dominant in this media. Therefore, the particles were aggregated and get settled down in the solvent. This phenomenon indicates the

Table 2. Grafting density of GPTMS/Imidazole on the surface of $G G S$ and $I G S$ particles.

\begin{tabular}{|c|c|c|c|c|c|}
\hline Sample code & $\begin{array}{c}\text { Weight loss at } \mathbf{3 0 0}^{\circ} \mathbf{C}, \\
\boldsymbol{W}_{\mathbf{3 0 0}}\end{array}$ & $\begin{array}{c}\text { Weight loss at } \mathbf{8 0 0}^{\circ} \mathbf{C}, \\
\boldsymbol{W}_{\mathbf{8 0 0}}\end{array}$ & $\begin{array}{c}\boldsymbol{W}_{\mathbf{3 0 0}}-\boldsymbol{W}_{\mathbf{8 0 0}} \\
{[\mathbf{w t} \mathbf{\%}]}\end{array}$ & $\begin{array}{c}\text { Grafting density } \\
{[\mathbf{w t} \mathbf{\%}]}\end{array}$ & $\begin{array}{c}\text { Grafting density } \\
{[\mathbf{m m o l} / \mathbf{g} \text { of } \boldsymbol{A S S}]}\end{array}$ \\
\hline$A S S$ & 89.0 & 82.0 & 7 & 0 & 0.00 \\
\hline$G G S$ & 92.0 & 76.0 & 16 & 9 & 0.80 \\
\hline$I G S$ & 90.6 & 72.6 & 18 & 2 & 2.67 \\
\hline
\end{tabular}




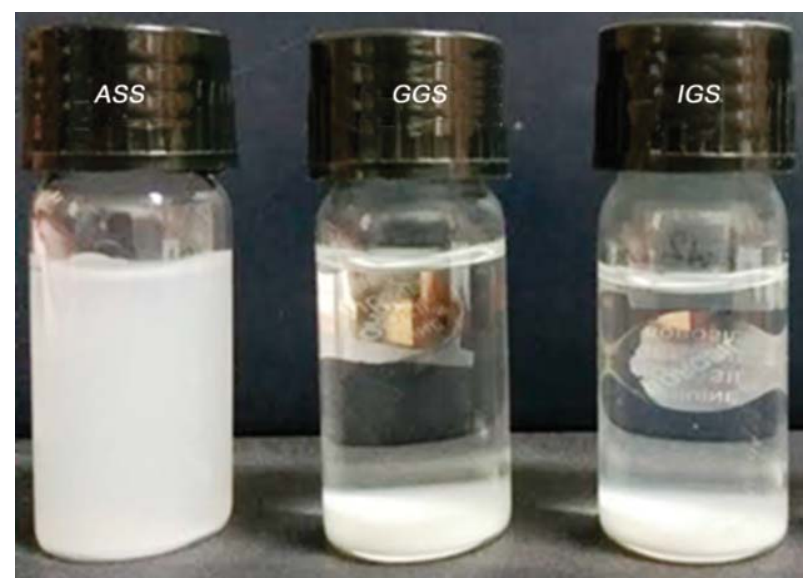

Figure 5. Photograph showing dispersion behavior of $A S S$, $G G S$, and $I G S$ in toluene.

resence of GPTMS and imidazole on the surface of $\mathrm{SiO}_{2}$ nanoparticles.

To better evidence, the epoxy silane and imidazole successfully grafted on $A S S, X P S$ measurements were carried out on $A S S, G G S$, and IGS, as shown in Figure 6. Through the surveyed spectra in Figure 6a, the five peaks corresponding to silicon signals at $\sim 150$, $\sim 100, \sim 530, \sim 283$, and $\sim 400 \mathrm{eV}$ represent the binding energies for Si2s, Si2p, O1s, N1s, and C1s, respectively [36]. For $G G S$, the C1s spectrum was divided into three different peaks attributed to binding energies for $\mathrm{C}-\mathrm{O}(283.48 \mathrm{eV}), \mathrm{C}-\mathrm{C}(284.40 \mathrm{eV})$, and $\mathrm{C}-\mathrm{O}-\mathrm{C}(287.34 \mathrm{eV})$ as shown in Figure $6 \mathrm{~b}$. However, the Si2p spectrum shows two different peaks attributed to binding energy for $\mathrm{Si}-\mathrm{O}-\mathrm{C}$ $(101.8 \mathrm{eV})$ and $\mathrm{Si}-\mathrm{O}-\mathrm{Si}(102 \mathrm{eV})$, as depicted in Figure $6 \mathrm{c}$. The peak of $\mathrm{C}-\mathrm{O}-\mathrm{C}, \mathrm{C}-\mathrm{C}$, and $\mathrm{C}-\mathrm{O}-\mathrm{C}$ reveals the presence of the GPTMS that grafted onto $A S S$, and the presence of $\mathrm{Si}-\mathrm{O}-\mathrm{C}$ and $\mathrm{Si}-\mathrm{O}-\mathrm{Si}$ implies that the hydroxyl group successfully transformed into epoxide group [37]. Similarly, For $I G S$, the $\mathrm{C} 1 \mathrm{~s}$ spectrum was divided into two different peaks attributed to the binding energies for $\mathrm{C}-\mathrm{O}$ $(282.05 \mathrm{eV})$ and $\mathrm{C}-\mathrm{C}(283.3 \mathrm{eV})$ as presented in Figure $6 \mathrm{~d}$. However, the N1s spectrum indicates two different peaks attributed to binding energy for $\mathrm{C}=\mathrm{N}$ $(399.7 \mathrm{eV})$ and $\mathrm{C}-\mathrm{N}(399 \mathrm{eV})$, as depicted in Figure $6 \mathrm{f}$. The peaks of $\mathrm{C}-\mathrm{O}$ and $\mathrm{C}-\mathrm{C}$ suggest the presence of multi-hydrocarbon chains of silane coupling agent and the presence of $\mathrm{C}=\mathrm{N}$ and $\mathrm{C}-\mathrm{N}$ further reveals the presence of pentacyclic imide group of imidazole [38].

\subsection{Cure behavior of neat epoxy and epoxy nanocomposites}

Non-isothermal $D S C$ was employed to examine the effect of with and without functionalized $\mathrm{SiO}_{2}$ nanoparticles on cure characteristics of the epoxy/amine
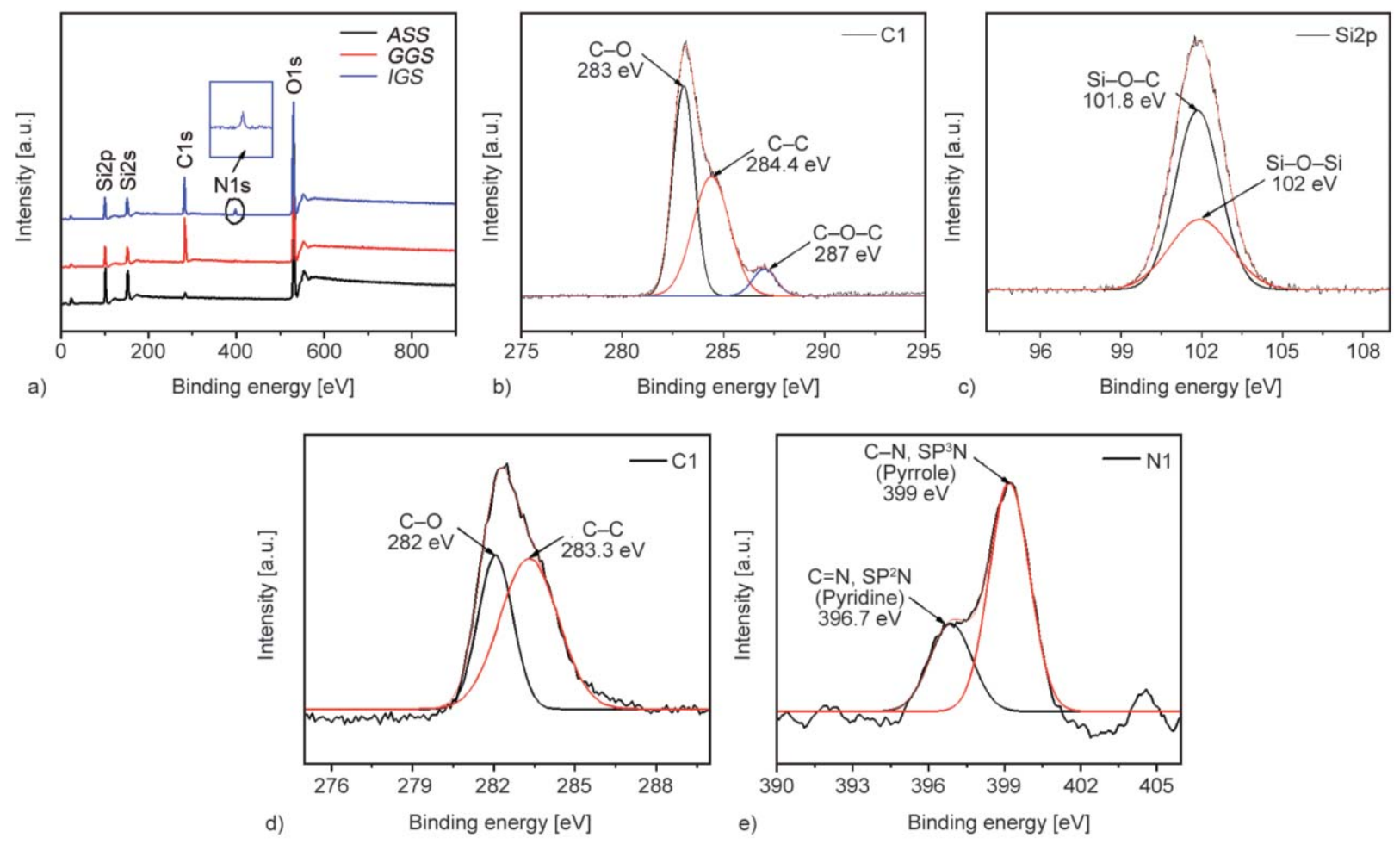

Figure 6. (a) X-ray-photoelectron spectroscopy (XPS) broad scans for $A S S, G G S$ and $I G S$ (b) Deconvoluted curve-fitting of $G G S$ for XPS core-level of C1s (c) Deconvoluted curve-fitting of $G G S$ for XPS core-level of Si2P (d) Deconvoluted curve-fitting of $I G S$ for XPS core-level of C1 (e) Deconvoluted curve-fitting of IGS for XPS core-level of N1. 
system. It is well-known that epoxy/amine curing reaction has an autocatalytic nature, whether three is a presence of nanoparticles or not. The facilitation of the epoxy ring-opening primarily evidences this autocatalysis with the help of $\mathrm{OH}$ groups formed in the course of curing reaction [39]. The reaction begins with dehydrogenation between the primary amine with the epoxide ring to form secondary amine. Then, the ring-opening of another epoxy monomer takes place by the secondary amine, and it goes on. It is also possible to have etherification side reactions through the reaction between $\mathrm{OH}$ groups generated during the crosslinking and epoxide groups [40]. For this purpose, the curing behavior of neat epoxy and its nanocomposites containing pristine $A S S, G G S$, and $I G S$ were studied at a heating rate, $\beta$ of $10^{\circ} \mathrm{C} \cdot \mathrm{min}^{-1}$, as shown in Figure 7. A single exothermic peak was observed in Figure 7 for all samples suggesting the unchanged cure reaction mechanism of epoxy due to the addition of bare or functionalized $\mathrm{SiO}_{2}$ nanoparticles.

This behavior indicates the fact that the epoxy/amine curing reaction underwent autocatalytic crosslinking

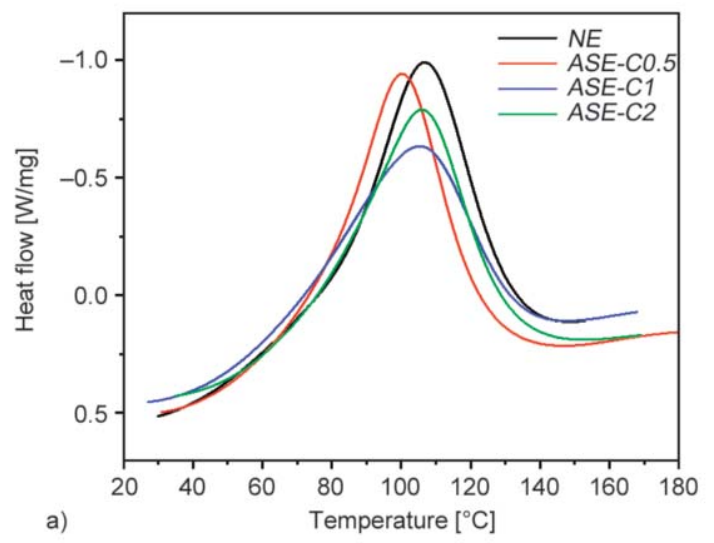

because of the facilitation of the epoxy ring-opening, which was intensified by $\mathrm{OH}$ groups. Under the completion of the curing, the exothermal peak temperature $\left(T_{\mathrm{p}}\right)$ is found maximum for a given temperature interval $(\Delta T)$. The total heat of reaction $\left(\Delta H_{\infty}\right)$ as calculated for neat epoxy and epoxy nanocomposite systems that was used to calculate $\Delta T^{*}, \Delta H^{*}$, and the Cure Index $(C I)$ is depicted in Table 3.

The $\Delta T, \Delta T^{*}, \Delta H^{*}$ and the $C I$ quantities are calculated by Equations (10)-(16):

$$
\begin{aligned}
& \Delta T=T_{\text {Endset }}-T_{\text {Onset }} \\
& \Delta H^{*}=\frac{\Delta H_{\mathrm{NC}}}{\Delta H_{\mathrm{NE}}} \\
& \Delta H^{*}=\frac{\Delta H_{\mathrm{NC}}}{\Delta H_{\mathrm{NE}}} \\
& C I=\Delta T^{*} \cdot \Delta H^{*} \\
& C I<\Delta T^{*} \equiv \text { Poor cure }
\end{aligned}
$$

$\Delta T^{*}<C I<\Delta H^{*} \equiv$ Excellant cure

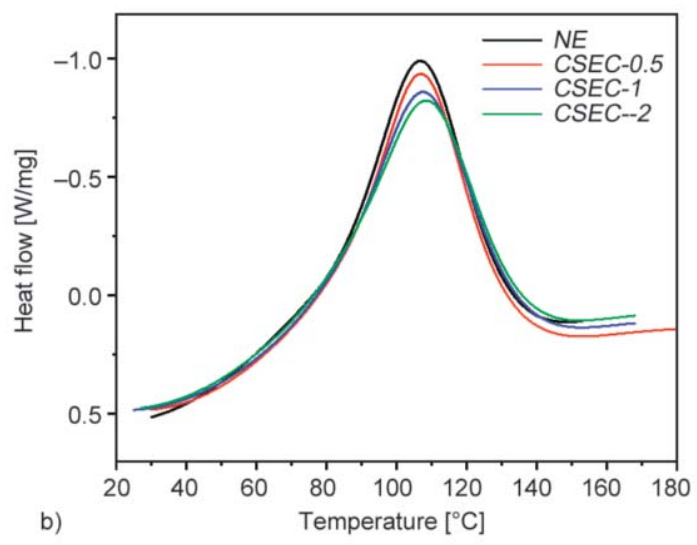

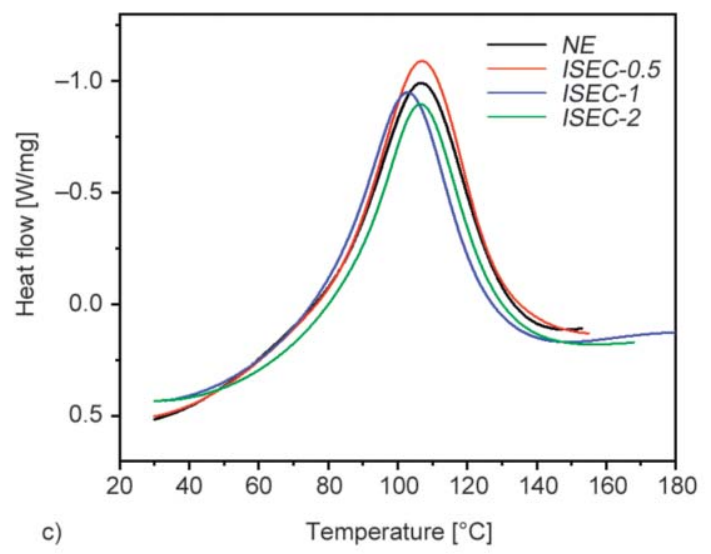

Figure 7. Non-isothermal DSC thermograms of NE and epoxy nanocomposites (a) ASS/epoxy (b) GGS/epoxy and (c) $I G S /$ epoxy nanocomposites at heating rates $(\beta)$ of $10^{\circ} \mathrm{C} \cdot \mathrm{min}^{-1}$. 
Table 3. The characteristic cure parameters and the cure Index in three different curing behavior of poor (red), Good (blue) and Excellent (green) of neat epoxy and epoxy nanocomposites as a function of $\beta=20^{\circ} \mathrm{C} / \mathrm{min}$ heating scan rates.

\begin{tabular}{|c|c|c|c|c|c|c|c|c|}
\hline $\begin{array}{c}\text { Samples } \\
\text { designation }\end{array}$ & $\begin{array}{l}T_{\text {Peak }} \\
{\left[{ }^{\circ} \mathbf{C}\right]}\end{array}$ & $\begin{array}{c}T_{\text {Onset }} \\
{\left[{ }^{\circ} \mathbf{C}\right]}\end{array}$ & $\begin{array}{c}T_{\text {Endset }} \\
{\left[{ }^{\circ} \mathbf{C}\right]}\end{array}$ & $\begin{array}{c}\Delta T \\
{\left[{ }^{\circ} \mathbf{C}\right]}\end{array}$ & $\begin{array}{l}\Delta \boldsymbol{H}_{\infty} \\
{[\mathbf{J} / \mathbf{g}]}\end{array}$ & $\Delta T^{*}$ & $\Delta H^{*}$ & $C I$ \\
\hline$N E$ & 107 & 30 & 153 & 123 & 363.6 & 1.00 & 1.00 & 1.00 \\
\hline ASEC-0.5 & 100 & 31 & 193 & 162 & 366.5 & 1.32 & 1.00 & 1.32 \\
\hline$A S E C-1$ & 105 & 27 & 168 & 141 & 314.4 & 1.14 & 0.84 & 0.96 \\
\hline$A S E C-2$ & 106 & 35 & 169 & 134 & 329.9 & 1.09 & 0.89 & 0.97 \\
\hline GSEC-0.5 & 107 & 30 & 187 & 157 & 370.0 & 1.27 & 1.02 & 1.29 \\
\hline GSEC-1 & 108 & 25 & 168 & 143 & 349.6 & 1.62 & 0.95 & 1.55 \\
\hline GSEC-2 & 108 & 27 & 168 & 141 & 343.6 & 1.14 & 0.94 & 1.07 \\
\hline ISEC-0.5 & 107 & 30 & 155 & 125 & 386.3 & 1.02 & 1.07 & 1.09 \\
\hline ISEC-1 & 103 & 33 & 183 & 150 & 343.5 & 1.22 & 0.94 & 1.14 \\
\hline ISEC-2 & 107 & 30 & 168 & 138 & 320.9 & 1.12 & 0.86 & 0.97 \\
\hline
\end{tabular}

$$
C I>\Delta H^{*} \equiv \text { Good cure }
$$

where $\Delta T_{\mathrm{NC}}$ and $\Delta T_{\mathrm{NE}}$ are the cure temperature intervals for the prepared epoxy nanocomposites and neat epoxy, respectively. In addition, $\Delta H_{\mathrm{NC}}$ and $\Delta H_{\mathrm{NE}}$ are the heat of cure for the prepared epoxy nanocomposites and neat epoxy, respectively, obtained from DSC analysis.

The effect of functionalized nanoparticles addition on the cure reaction of epoxy resin can be interpreted by tracking the event between the acceleration roles of functional groups attached on the surface of $\mathrm{SiO}_{2}$ particles from one side and the retardation effect caused by the steric hindrance of nanoparticles from the other side as contradictive effects controlling over crosslinking [41]. From the molecular perspective, the addition of nanofillers to the epoxy system makes mobility restriction of the reactive species can lead to an increase in the viscosity of the system. In this sense, one may expect a hindrance to cure reaction of epoxy by the introduction of pristine $A S S$, particularly by increasing the $A S S$ content from 0.5 to $2 \mathrm{wt} \%$ except for $0.5 \mathrm{wt} \%$ due to proper dispersion. For epoxy nanocomposites containing $I G S$ nanoparticles, a similar trend is expected at $2 \mathrm{wt} \%$. However, nanocomposites at $0.5 \mathrm{wt} \%$ of $I G S$ shows good cure behavior and excellent cure behavior at $1 \mathrm{wt} \%$, respectively. The acceleration in curing of epoxy nanocomposites at $0.5 \mathrm{wt} \%$ of IGS demonstrating the presence of imide group on $I G S$ with effective dispersion participating cure reaction in epoxy ringopening. In contrast, epoxy silane functionalized $\mathrm{SiO}_{2}$ nanoparticles $(G G S)$ may accelerate the curing reaction of epoxy at $0.5 \mathrm{wt} \%$, indicating good cure behavior $\left(C I>\Delta T^{*}\right)$ and excellent cure behavior at
1 and $2 \mathrm{wt} \%$ as satisfying the condition of $\Delta T^{*}<$ $C I<\Delta H^{*}$. This attributed to the presence of highly reactive epoxide groups on the surface of $G G S$, participating in epoxy ring-opening resulting improvement in cure quality of GGS/epoxy nanocomposites at $0.5 \mathrm{wt} \%$. The effect of adding functionalized silica nanoparticles on the crosslinking potential of epoxy system can be simply studied by the use of $C I$ in terms of nanofiller contents and surface chemistry at a fixed heating rate $\left(20^{\circ} \mathrm{C} / \mathrm{min}\right)$, as represented in Figure 8. It should be mentioned that $C I$ was introduced and examined in qualitative analysis of cure in epoxy systems containing $A S S, G G S$ and $I G S$. The bar diagrams at the top of Figure 8 demonstrate three types of cure, i.e., poor (red), excellent (green), and good cure (blue), in terms of the $C I$. As the variation of $C I$ suggests, all possible cases take place in the

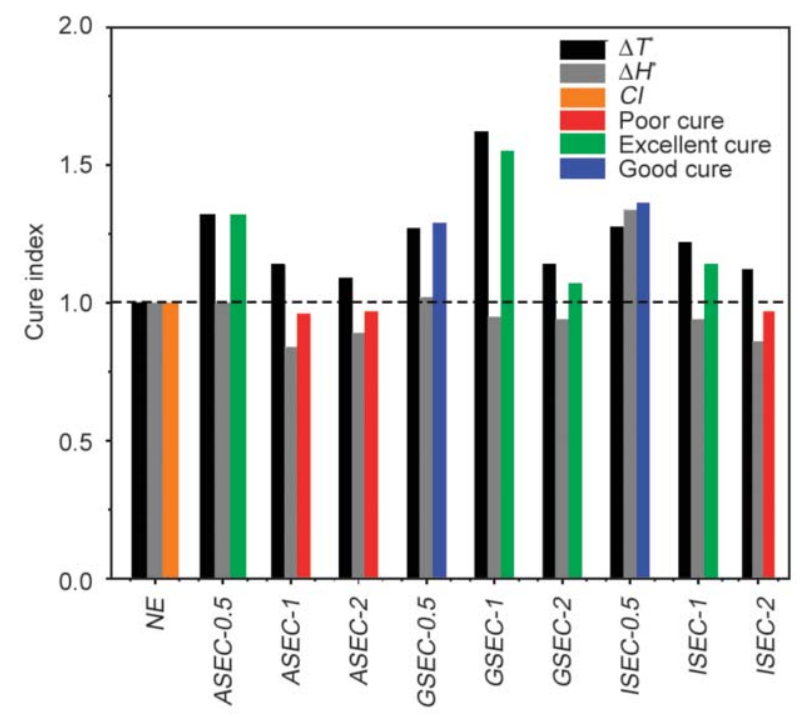

Figure 8. Typical cure behavior in terms of $\Delta T^{*}, \Delta H^{*}$, and $C I$ for the prepared neat epoxy and epoxy nanocomposites containing $A S S, G G S$, and $I G S$ at a heating rate of $10^{\circ} \mathrm{C} \cdot \mathrm{min}^{-1}$. 
course of curing reactions in nanocomposites cured at $10^{\circ} \mathrm{C} \cdot \mathrm{min}^{-1}$ heating rate depending on the nature and amount of nanoparticles. Individual use of $A S S$ and $I G S$ at higher $\mathrm{wt} \%$ leads to poor cure situation for ASEC-1, ASEC-2, and ISEC-2, whereas the use of $G G S$ and $I G S$ nanoparticles at lower content causes a little increase in crosslinking featured by good cure behavior associated with GSEC-0.5 and ISEC-0.5 composites. However, at higher contents of GGS and IGS shows an excellent cure for GSEC-1, $G S E C-2$, and $I S E C-1$, respectively. Hence, from the above discussion, it concludes the high reactivity of $G G S$ and $I G S$ at lower particle contents have the potential for crosslinking network formation in the epoxy resin, as illustrated in Figure 8.

\subsection{Tensile properties of epoxy nanocomposites}

The tensile properties of nanocomposites with varying content $(0-2 \mathrm{wt} \%)$ of $A S S, G G S$, and $I G S$ are presented in Figure 9, and for comparison, the values are depicted in Table 4. The tensile strength, tensile modulus, and modulus of toughness for neat epoxy are found as $48,1.05$, and $1.91 \mathrm{MJ} \cdot \mathrm{m}^{-3}$, respectively. Maximum tensile strength and modulus of toughness are observed for $A S E C$ having $0.5 \mathrm{wt} \%$ of $A S S$ and the improvement is $\sim 58$ and $\sim 100 \%$ compared to that of the $N E$. The tensile modulus maximizes to $\sim 17 \%$ compared to $N E$ at higher particle content for $A S E C$. In contrast, GSEC gives maximum increment in tensile strength and modulus of toughness by $\sim 65 \%$ (79 MPa), and $\sim 272 \%\left(7.10 \mathrm{MJ} \cdot \mathrm{m}^{-3}\right)$ compared to the $N E$ with $0.5 \mathrm{wt} \%$ of $G G S$ addition. However, the tensile modulus enhances by $\sim 21 \%(1.27 \mathrm{GPa})$ when

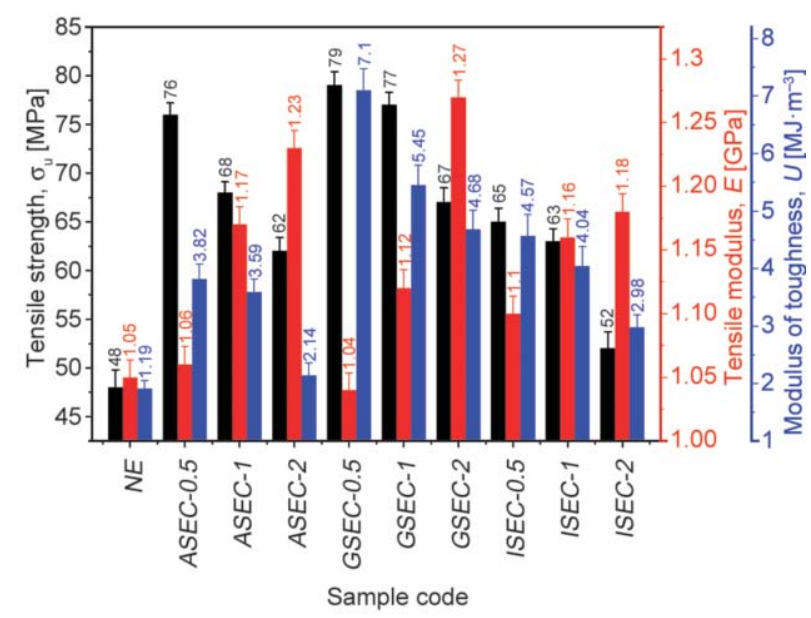

Figure 9. Tensile properties variation of epoxy nanocomposites with varying contents of $A S S, G G S$, and $I G S$ nanoparticles.
Table 4. Tensile properties of epoxy nanocomposites.

\begin{tabular}{|l|c|c|c|}
\hline Sample code & $\begin{array}{c}\text { Tensile } \\
\text { strength } \\
{[\mathbf{M P a}]}\end{array}$ & $\begin{array}{c}\text { Tensile } \\
\text { modulus } \\
{[\mathbf{M P a}]}\end{array}$ & $\begin{array}{c}\text { Modulus of } \\
\text { toughness } \\
{\left[\mathbf{M J} \cdot \mathbf{m}^{-3}\right]}\end{array}$ \\
\hline$N E$ & $48 \pm 1.8$ & $1058 \pm 13.8$ & $1.91 \pm 0.14$ \\
\hline ASEC-0.5 & $76 \pm 1.2$ & $1060 \pm 14.2$ & $3.82 \pm 0.26$ \\
\hline ASEC-1 & $68 \pm 1.6$ & $1170 \pm 13.9$ & $3.59 \pm 0.23$ \\
\hline ASEC-2 & $65 \pm 1.4$ & $1230 \pm 13.7$ & $2.14 \pm 0.22$ \\
\hline GSEC- 0.5 & $79 \pm 1.5$ & $1040 \pm 13.4$ & $7.10 \pm 0.38$ \\
\hline GSEC-1 & $77 \pm 1.3$ & $1120 \pm 14.7$ & $5.45 \pm 0.35$ \\
\hline GSEC-2 & $67 \pm 1.5$ & $1270 \pm 13.1$ & $4.68 \pm 0.33$ \\
\hline ISEC-0.5 & $65 \pm 1.4$ & $1100 \pm 13.7$ & $4.57 \pm 0.37$ \\
\hline ISEC-1 & $63 \pm 1.3$ & $1160 \pm 14.3$ & $4.04 \pm 0.34$ \\
\hline ISEC-2 & $52 \pm 1.7$ & $1180 \pm 14.1$ & $2.98 \pm 0.22$ \\
\hline
\end{tabular}

$2 \mathrm{wt} \%$ of $G G S$ was added. This enhancement is believed from the crosslinking capability of $G G S$ with the epoxy system, which is expected to tailor the interfacial adhesion properties [42].

Moreover, the hydrophobic GGS covalently bonded with the epoxy network might help in the generation of the efficient interface at their juncture to modulate interfacial stiffness. This is also evident from the results obtained in the cure behavior of epoxy nanocomposites. It was found that adding $G G S$ into epoxy at $0.5 \mathrm{wt} \%$ has a good cure quality of nanocomposites in comparison to other composite systems. This is because the presence of the epoxide group on the surface of GGS leads to accelerate the curing reaction with epoxy ring-opening during polymerization that formed a strong crosslinking network and promotes the homogeneous distribution of nanofillers. Since the $G G S$ nanoparticles possess a higher $C I$ than the other composite systems, tensile stress exists at the interface of $G G S$ and the epoxy matrix. This caused an additional stress transfer from $G G S$ to the epoxy matrix when the sample was under loading [43]. Therefore, the GSEC-0.5 composite shows a significant enhancement in mechanical properties as compared to other systems of composites. Further, the maximum increment in tensile strength by $\sim 35 \%$ (65 MPa), modulus of toughness by $\sim 139 \%$ $\left(4.57 \mathrm{MJ} \cdot \mathrm{m}^{-3}\right)$ is observed for IGSEC-0.5, while the tensile modulus is found enhanced by $\sim 12 \%$ $(\sim 1.18 \mathrm{GPa})$ for IGSEC-2, as compared to $N E$. In comparison to GSEC, ISEC showed inferior mechanical properties might be due to the presence of imide functional group on $I G S$ at high contents that may influence the crosslinking and close packing of the polymer chains, which negatively affected the mechanical properties [44]. By increasing nanoparticle 
content $(>1 \mathrm{wt} \%)$, the tensile strength, and modulus of toughness start decreasing. This may be attributed to the plasticizing effect of excessive silane on $G G S$ particles inhibiting the curing process and retard the crosslinking [45]. Afzal et al. [46] reported that epoxy functionalization of silica nanoparticles not only improves their distribution in the epoxy polymer but simultaneously enhances the tensile strength $(\sim 60 \%)$, Young's modulus $(\sim 85 \%)$ and tensile toughness $(\sim 56 \%)$ even at $15 \mathrm{wt} \%$ due to stronger interfacial interactions.

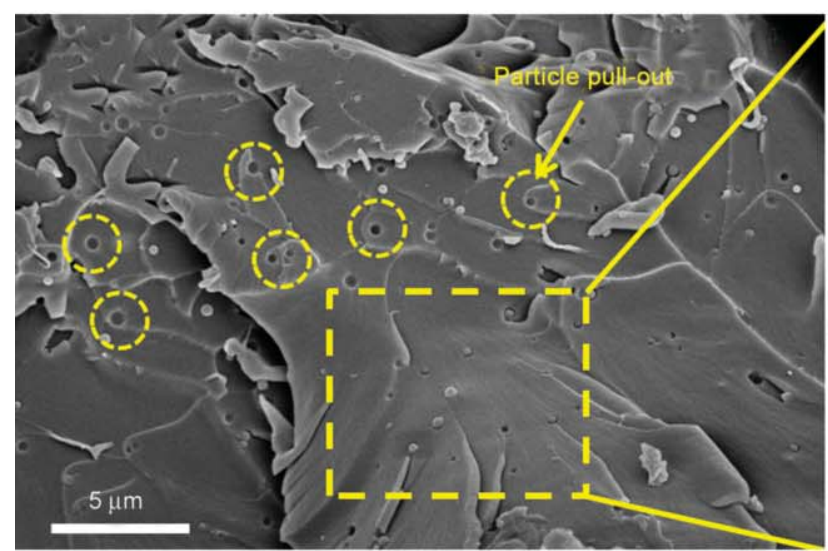

a)

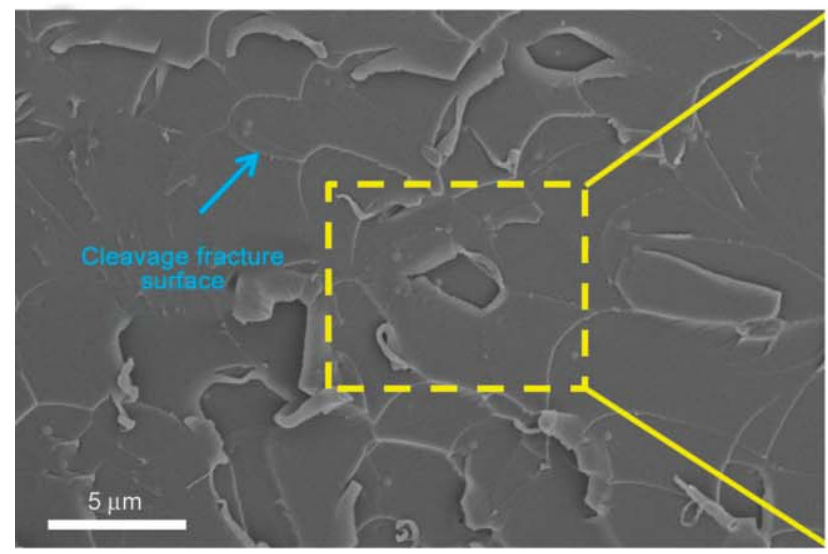

c)

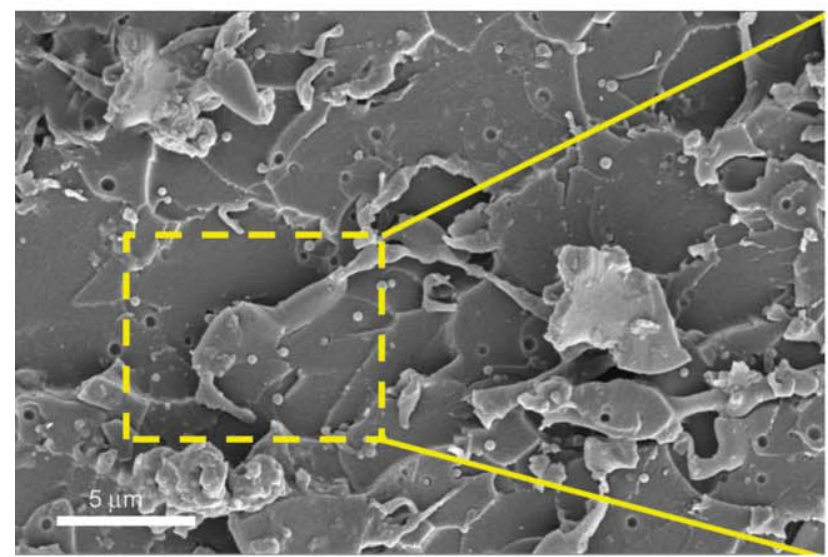

e)

\subsection{The failure mechanism of epoxy nanocomposites}

The fractography analysis of the tensile fracture surface of NE, ASEC, GSEC, and ISEC was performed under FESEM to demonstrate the various failure mechanisms associated with it, as shown in Figure 10. The fracture surface of the composite containing $0.5 \mathrm{wt} \%$ of $A S S$ exposes the poorly adhered $A S S$ particles and voids because of particle pullout, as can be seen in Figure 10a. This poorly adhered particle leads to hindrance in the crosslinking of the

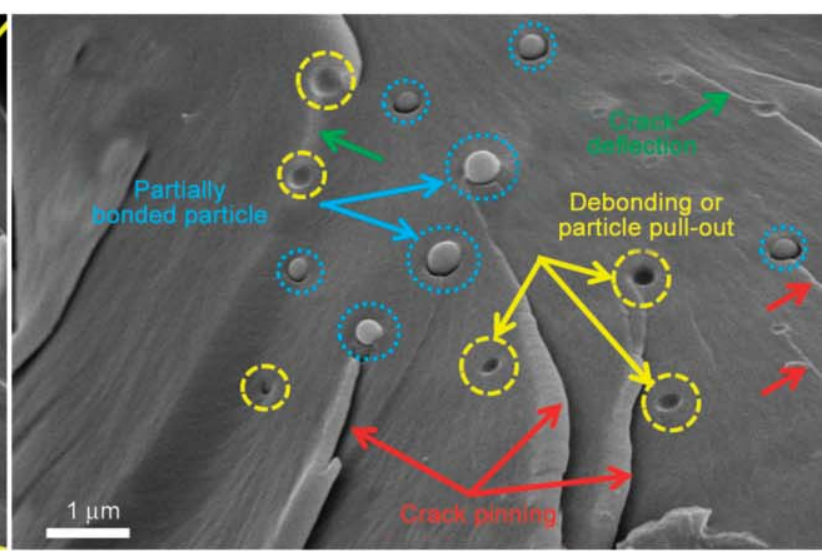

b)

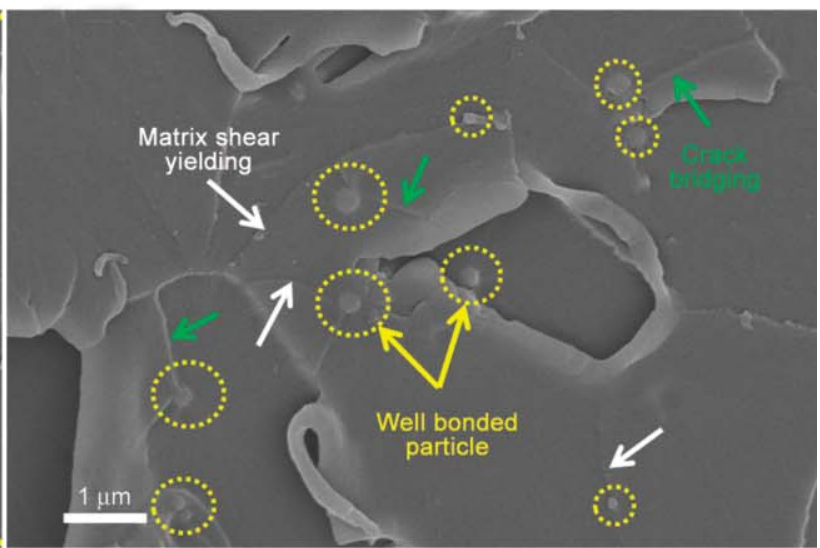

d)

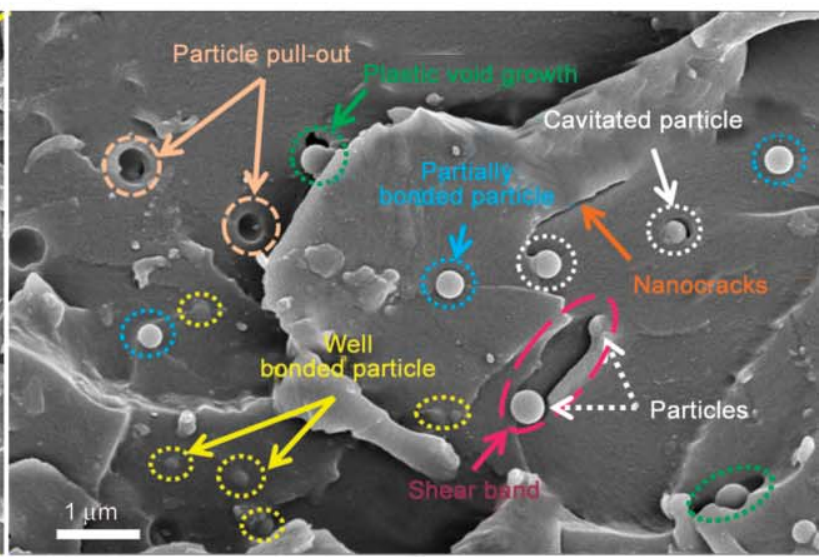

f)

Figure 10. Microscopic tensile fracture surfaces of epoxy nanocomposites filled with $0.5 \mathrm{wt} \%$ of; (a, b) $A S S$ (c, d) $G G S$ and (e, f) $I G S$. 
epoxy network [47]. Micrograph at higher magnification, Figure 10b further confirms particle pullout and the formation of nano-level voids (circumscribed by circles in Figure 10b). Thus, the resistance to fracture is created by plastic void growth around the partially adhered nanoparticles and the void formation because of the particle pullout.

These features result in the creation of the crack tail at the vicinity of partially adhered $A S S$ or the voids resulting in crack arrest due to pinning and later generates the cleavage surfaces [48]. The cleavage surfaces are clear and planed with a minimum amount of thin lines representing microcracks. For composites $G S E C$, a relatively rougher fracture surface indicated by the presence of a large number of cleavage surface is observed, as can be seen in Figure 10c. In contrast to $A S E C$, the fracture surface does not reveal unadhered nanoparticles or voids, which is believed to the good adhesion of the GGS nanoparticles within the matrix. Higher magnification fractography image of GSEC, Figure 10d reveals the well-bonded nanoparticle (yellow encircled in Figure 10d) within the cleavage surfaces. The cleavage surface fracture borders are far away from the well-embedded GGS nanoparticles. Besides, places are found wherein thin matrix shear yielding marks (marked with white arrows in Figure 10d are found where the crack is pinned because of the tailored interfacial stiffness at the juncture of $G G S$ and the matrix. This behavior endorses the modulation in the crosslinking density of the epoxy network due to GGS. Nanoparticles covered by the layer of the epoxy matrix indicate that the cracks might have propagated through the polymer matrix above or below the poles of the nanoparticles, resulting in more energy consumption due to crack deflection. Crack bridging (marked by the green arrow) is also observed, which occurs when a crack propagates and encounters a particle right in front of its path [49]. This type of mechanism can only be observed when there is a strong nanoparticle-matrix interface. A strong interface can bridge the crack and not allow it to propagate in the surrounding region further. For $I S E C$, a comparatively rough fracture surface is observed, as depicted in Figure 10e. The cleavage fracture surface is surrounded by a large number of small fracture sites leading to the enhancement in fracture surface roughness. Mixed fracture features, such as particle pullout, partially bonded particles, and well-bonded particles, are found from the high magnification FESEM fracture surface image in Figure 10f. The IGS nanoparticles which are unable to create an interfacial network with the epoxy matrix pops up during fracture leaving behind particle pull out holes. Partially adhered IGS creates plastic void growth around them that enhances the fracture resistance [50].

\subsection{Interface and microstructure in epoxy nanocomposites}

In order to further understand the influence of $A S S$, $G G S$, and $I G S$ on the particle/matrix interface properties, the above failure mechanism postulated from the fracture surface is explained schematically and presented in Figure 11. The fracture surface of neat epoxy is represented by matrix micro-cracking depicting brittle failure under the tensile loading. For $A S E C$, particle pullout and plastic void growth around the nanoparticles are shown that enhance the failure resistance. However, the scheme for the GSEC represents a strong particle-matrix interface leading to the formation of craze lines within the matrix producing crack bridging effect through the poles of $G G S$ particles. This crack bridging effect is expected due to the good wetting of $G G S$, which further provides strong particles-matrix interaction through covalent bonding.

Hence, the out of plane divergence of the crack path facilitates the generation of a tortuous fracture surface [51]. A mixed-mode failure mechanism is shown for ISEC. This mixed-mode failure includes particle pullout, well-bonded particles, and the partially bonded particles and formation of nanovoids around IGS nanoparticles leading to poor particle-matrix adhesion and thus responsible for the lower enhancement in tensile properties as compare to GSEC composites. This behavior is also supported by the FESEM tensile fracture surface of composites filled with $0.5 \mathrm{wt} \%$ of $A S S, G G S$, and $I G S$ at high magnification, as presented in Figure 12. Figure 12a shows the smooth and finer river-like structure, which indicates matrix microcracking resulting in brittle failure of $N E$. The fracture surface of composites having $0.5 \mathrm{wt} \%$ of ASS shows a combination of particle pullout and partially bonded particle failure mechanism, as depicted in Figure 12b. However, Figure 12c shows well-embedded non-aggregated $G G S$ nanoparticles in the epoxy matrix that lead to the crack propagation around the particles. This indicates a better interfacial stiffness of the $G G S / E P-0.5$ system. Consequently, it increases the load transferring ability of $G G S$ from the matrix 
to nanoparticles. For the $I G S / E P-0.5$ nanocomposites, the high magnification FESEM image demonstrates a mixed-mode of failure. The failure includes particle pullout, partially bonded particles, and well-bonded

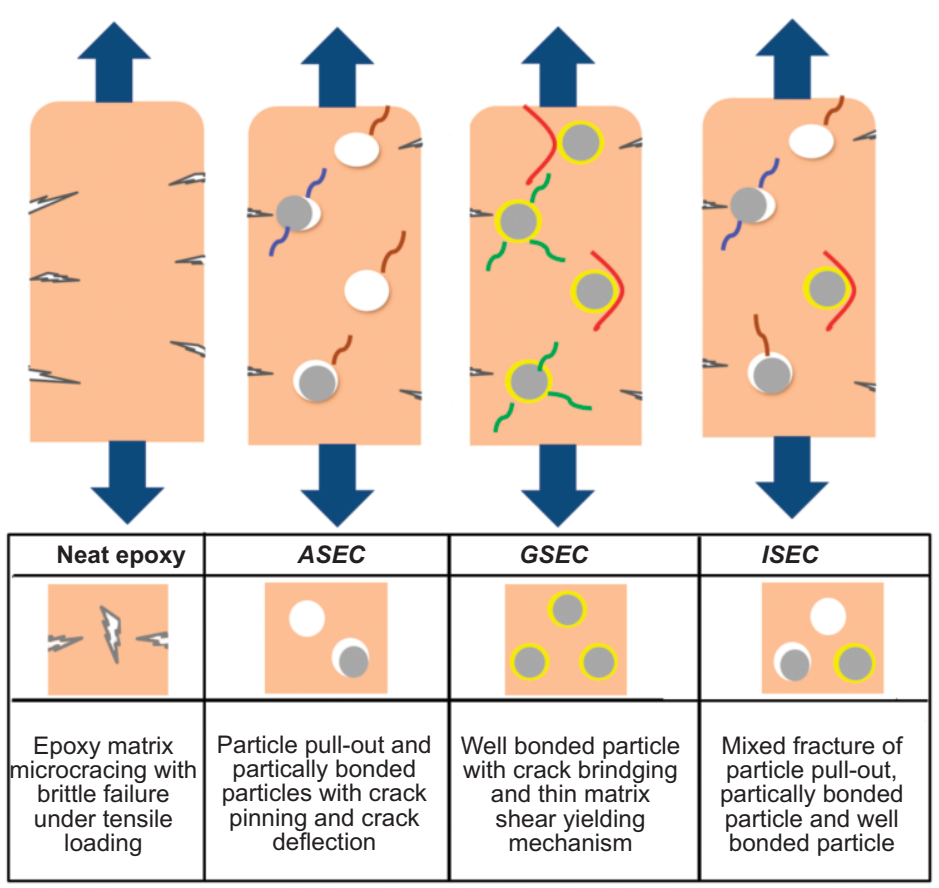

Figure 11. Schematic representations for major failure mechanisms of epoxy resin containing $A S S, G G S$, and $I G S$ nanoparticles under tension test.
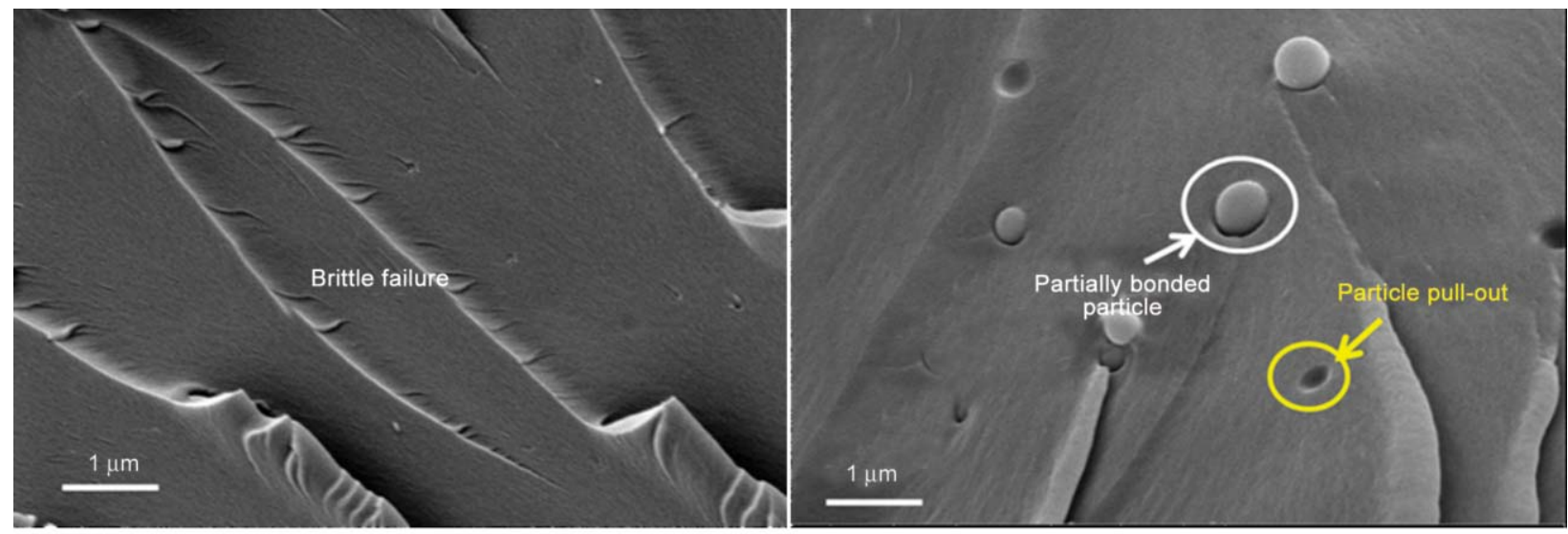

a)

b)
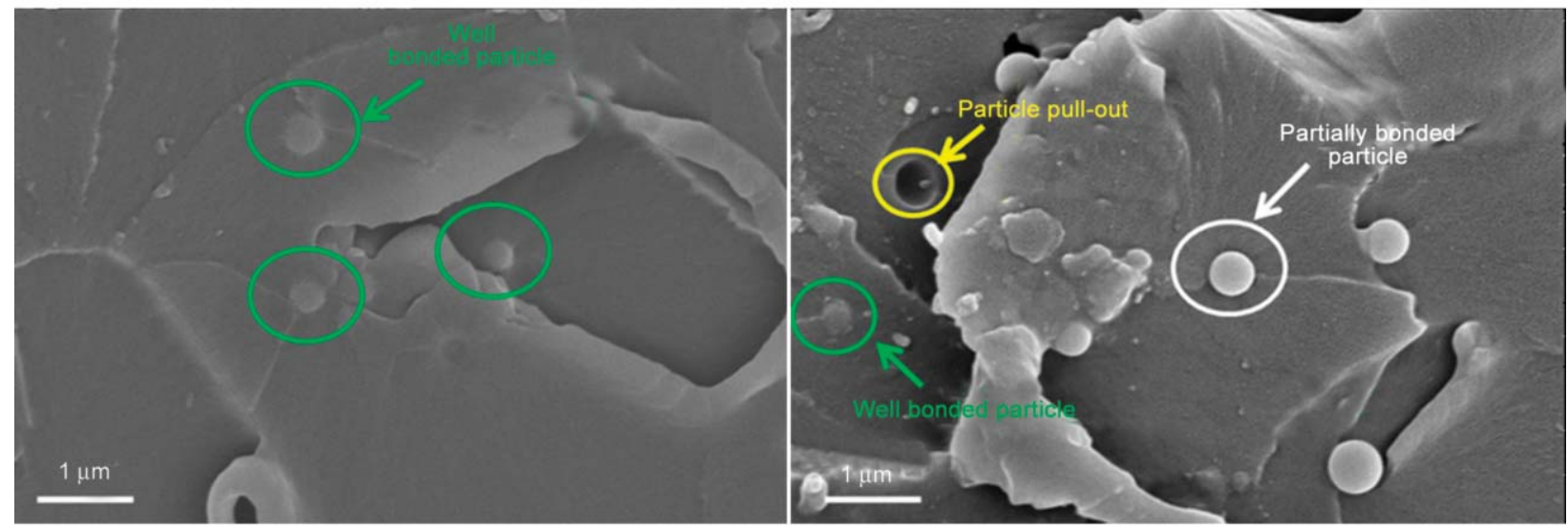

c)

d)

Figure 12. Micrograph at high magnification of tensile fracture surfaces of (a) $N E$ and nanocomposites filled with $0.5 \mathrm{wt} \%$ of (b) $A S S$ (c) GGS and (d) IGS. 
particles, as shown in Figure 12c. Such aggregates of $I G S$ may hinder the modification of the interfacial stiffness and can create stress concentration zones during the fracture process, impairing the stress transferability of the matrix.

\subsection{Flexural properties of epoxy nanocomposites}

This test was performed to investigate the influences of functionalized silica nanoparticles on the flexural properties of the composites. The flexural properties of nanocomposites against varying content $(0-2 \mathrm{wt} \%)$ of $A S S, G G S$, and $I G S$ nanoparticles, along with neat epoxy, are shown in Figure 13. The flexural strength, flexural modulus, and work of flexural of the neat epoxy are found as $\sim 113 \mathrm{MPa}, \sim 2.35 \mathrm{GPa}$, and $\sim 9.0 \mathrm{MJ} / \mathrm{m}^{2}$, respectively. For composites $(A S E C)$ added with $0.5 \mathrm{wt} \%$ ASS nanoparticles, the maximum flexural strength, flexural modulus, and work of flexural of $\sim 130 \mathrm{MPa}, \sim 3.33 \mathrm{GPa}$, and $\sim 8.51 \mathrm{MJ} / \mathrm{m}^{2}$, respectively, are found. This result leads $\sim 15$ and $42 \%$ increment and $\sim 5 \%$ reduction in flexural strength, flexural modulus, and work of flexural, respectively, compared to the NE. In contrast, composites (GSEC) added with $0.5 \mathrm{wt} \% G G S$ nanoparticles showed the maximum flexural strength, flexural modulus, and work of flexural of $\sim 168 \mathrm{MPa}, \sim 3.53 \mathrm{GPa}$, and $\sim 13.28 \mathrm{MJ} / \mathrm{m}^{2}$, respectively.

This maximum improvement was $\sim 48, \sim 50$, and $\sim 47 \%$ in flexural strength, flexural modulus, and work of flexural, respectively, compared to $N E$. For composites, (ISEC) added with $0.5 \mathrm{wt} \%$ IGS nanoparticles, the maximum flexural strength, flexural modulus, and work of flexural of $\sim 129 \mathrm{MPa}$,

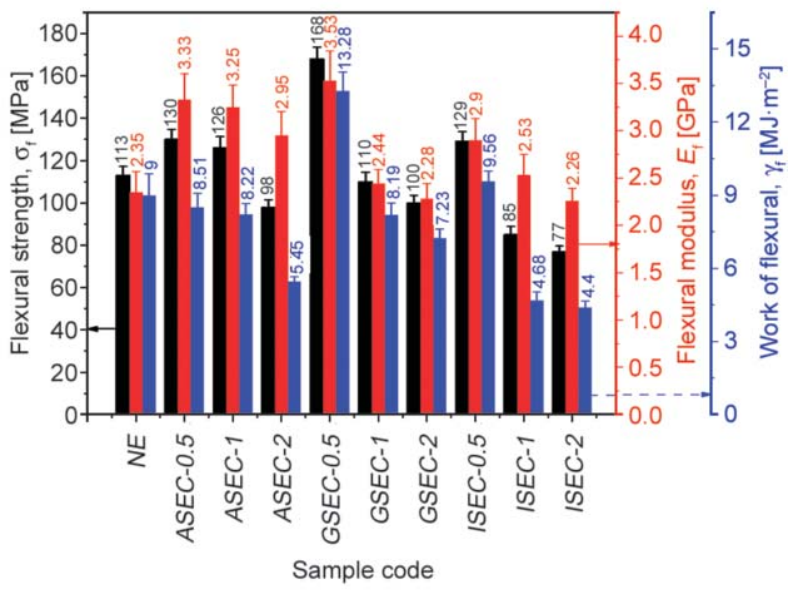

Figure 13. Flexural properties of epoxy nanocomposites with varying contents of $A S S, G G S$ and $I G S$ nanoparticles. $\sim 2.90 \mathrm{GPa}$, and $\sim 9.56 \mathrm{MJ} / \mathrm{m}^{2}$, respectively, are found. This leads to $\sim 14, \sim 23, \sim 6 \%$ enhancement compare to $N E$, respectively. The maximum enhancement in flexural properties due to the $G G S$ addition in the composites is due to the better distribution of $G G S$ and well bonding with the epoxy network. With the further addition of nanoparticles, ineffective dispersion of nanoparticle and ineffective stiffness modulation ability at the juncture of the agglomerates and the epoxy network might decrease the flexural performance of the composites [52]. The rigid fillers commonly increase the modulus (stiffness), but on the other side, they may have a catastrophic effect on the flexural strength. In reinforced polymers, it is commonly observed that the flexural strengths are higher than that of tensile strengths. Under a flexural loading situation, the fillers aid the load-bearing capability instead of acting as stress raisers, as is the case of tensile loading. This load-bearing capability is because the compressive stress tends to close the cracks and flaws that are perpendicular to the applied load, opposite to the crack opening mechanism occurring in a tensile strength situation [53].

\subsection{Fracture toughness of the nanocomposites}

The effect of adding functionalized silica on fracture toughness $\left(K_{1 \mathrm{C}}\right)$ and fracture energy $\left(G_{1 \mathrm{C}}\right)$ was studied using 3-point single-edge-notch bending (SENB) tests. The fracture properties of epoxy composites containing varying content $(0.5,1$, and $2 \mathrm{wt} \%)$ of $A S S, G G S$, and $I G S$ nanoparticles are presented in Figure 14.

The value of $K_{1 \mathrm{C}}$ and $G_{1 \mathrm{C}}$ for $N E$ is found as $\sim 0.70 \mathrm{MPa} \cdot \mathrm{m}^{0.5}$ and $\sim 407 \mathrm{~J} \cdot \mathrm{m}^{-2}$, respectively. The $K_{1 \mathrm{C}}$ and $G_{1 \mathrm{C}}$ for $A S E C$ are found a maximum at $0.5 \mathrm{wt} \%$ of $A S S$ addition of $\sim 1.08 \mathrm{MPa} \cdot \mathrm{m}^{0.5}$ and $\sim 970 \mathrm{~J} \cdot \mathrm{m}^{-2}$. In contrast, composites embedded with $0.5 \mathrm{wt} \%$ of $G G S$ showed significant enhancement in fracture toughness and fracture energy. The fracture toughness $\left(K_{1 \mathrm{C}}\right)$ and the fracture energy $\left(G_{1 \mathrm{C}}\right)$ of GSEC are $\sim 1.38 \mathrm{MPa} \cdot \mathrm{m}^{0.5}$ and $\sim 1612 \mathrm{~kJ} \cdot \mathrm{m}^{-2}$, which are $\sim 97$ and $\sim 296 \%$ higher than that of $N E$ respectively. For composite (ISEC) added with $0.5 \mathrm{wt} \%$ of $I G S$, the maximum increase in $K_{1 \mathrm{C}}$, and $G_{1 \mathrm{C}}$ are observed lesser than GSEC. We found $\sim 54 \%$ $\left(1.08 \mathrm{MPa} \cdot \mathrm{m}^{0.5}\right)$ and $\sim 132 \%\left(944 \mathrm{~J} \cdot \mathrm{m}^{-2}\right)$ increment than that of the NE, but better than the ASEC system. Further addition of $I G S$ nanoparticles more than $2 \mathrm{wt} \%$ diminishes the $K_{1 \mathrm{C}}$ and $G_{1 \mathrm{C}}$ value. For higher nanoparticle content ( $2 \mathrm{wt} \%$ ), the $K_{1 \mathrm{C}}$ and $G_{1 \mathrm{C}}$ 


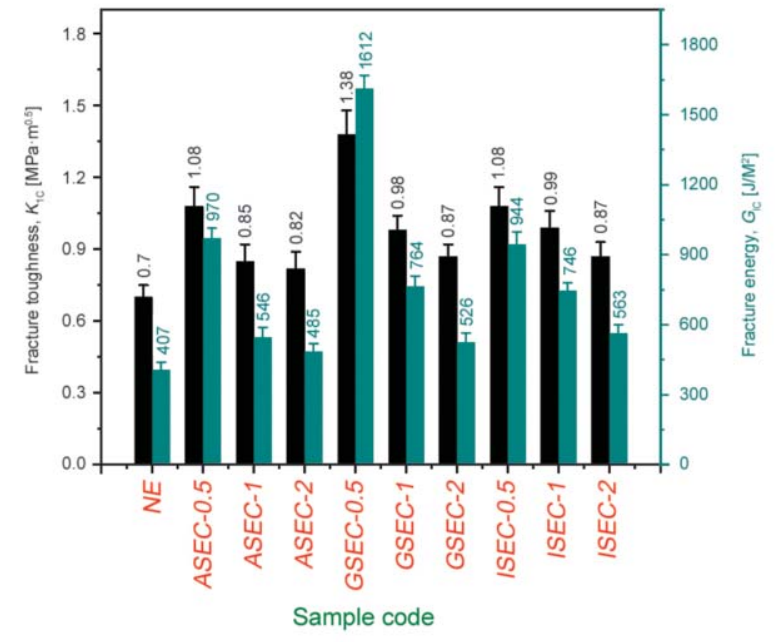

Figure 14. Fracture properties of composites with varying contents of $A S S, G G S$, and $I G S$ nanoparticles.

reduce to $\sim 0.87 \mathrm{MPa} \cdot \mathrm{m}^{0.5}$ and $\sim 563 \mathrm{~J} \cdot \mathrm{m}^{-2}$, which is found still higher than that of neat epoxy. A similar trend of $K_{1 \mathrm{C}}$ and $G_{1 \mathrm{C}}$ value is observed in the case of $A S E C$ and GSEC nanocomposites at $2 \mathrm{wt} \%$ filler contents. To achieve the same enhancement ( $\sim 24$ and $\sim 38 \%$ ) in $K_{1 \mathrm{C}}$, the required amount [wt $\%$ ] of nanoclay and alumina nanoparticles in the epoxy matrix is quite high [54]. In comparison to other reported work on using functionalized silica nanoparticles, we found the present system offers better resistance to fracture. In the case of ISEC composites, the clustering of nanoparticles might aids in diminishing the interfacial area at $\mathrm{SiO}_{2}$ epoxy matrix interface and hence, reduces the properties. The clustering leads to poor interaction between the $I G S$ and epoxy matrix, causing a detrimental effect on the fracture properties [54]. This could also be explained by correlation with the curing behavior of epoxy nanocomposites. The different particle addition has different cure behavior. As the results obtained from the non-isothermal DSC cure study, the GSEC- 0.5 composite has a good quality of cure in comparison to other composite systems. This might be attributed to the difference in the cure index $(C I)$ quantity for different composites systems. The results obtained from the cure qualities of different composite shows the GSEC-0.5 has the highest quality of cure in comparison to other composite systems even at higher particle contents due to satisfy the condition of $\Delta T^{*}<C I<\Delta H^{*}$. This attributed to $G G S$ has the potential to form a strong crosslinking network during polymerization, even at higher particle content [55]. This attributes the improvement in interfacial property between the $G G S$ and the epoxy matrix, and this leads to a significant enhancement in fracture property of GSEC-0.5 composites as compared to the other system. However, in the case of $A S S$ and $I G S$, the hydroxyl and imide functional groups may hinder the mobility of polymer chain at higher contents which lead to either poor quality or just the excellent cure condition is satisfied, lead to poor fracture behaviour as compare to GSEC.

The possible failure modes and toughening mechanism for ASEC, GSEC, and ISEC system under mode-I fracture are presented in Figure 15. In the scheme, the fracture plane of the composites system is subjected to both tensile as well as compressive load. The region of nanocomposites under the action of compressive load bear local crushing, and at the same time, the tensile region tended to initiate the crack. However, the local stress concentration site mainly originates at the notched or pre-crack region that leads to final fracture [56]. In the present study, the composite modified with $A S S$ particles produces voids and hemispherical cavities around the particles due to particle debonding within the matrix. The fracture toughness of nanocomposites was significantly influenced by the presence of the well-bonded $G G S$ in the epoxy network.

The enhancement of the fracture toughness behaviour justifies the formation of a fracture-toughening zone around the well-bonded GGS nanoparticles within the matrix. Crack propagation around the pole of the well-bonded nanoparticle is expected due to the creation of the toughening zone resulting in more energy absorption. Hence, the crack propagation is slowed due to the resistance offered by the volume energy of the system and delays the final fracture for GSEC [57]. For ISEC, because of the presence of partially adhered nanoparticles, the well-bonded $I G S$ nanoparticles do not offer resistance to crack propagation. The presence of poorly adhered IGS nanoparticles eases the crack to move without any obstacle leaving behind voids and hemispherical holes. So, this additional formation of the toughening zone produced by $G G S$ nanoparticles at lower content $(0.5 \mathrm{wt} \%)$ is also supported by the results obtained from the tensile fracture surface and the fracture property of $G G S /$ epoxy nanocomposites.

\section{Conclusions}

The functionalization of as-synthesized silica (ASS) with GPTMS/imidazole moieties was performed using the post-synthesized grafting route. The presence of 


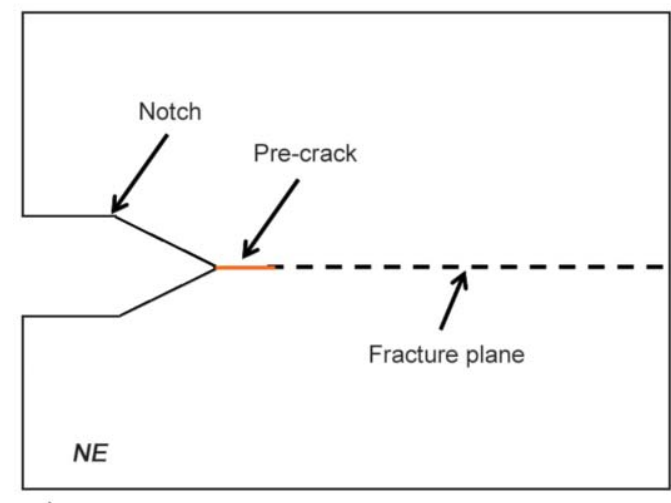

a)

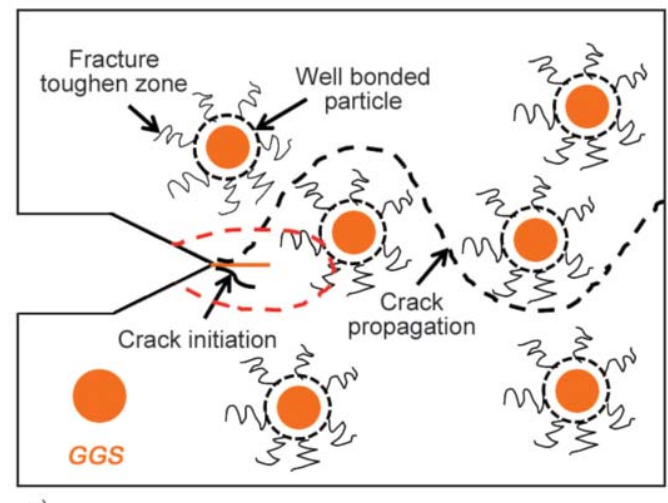

c)

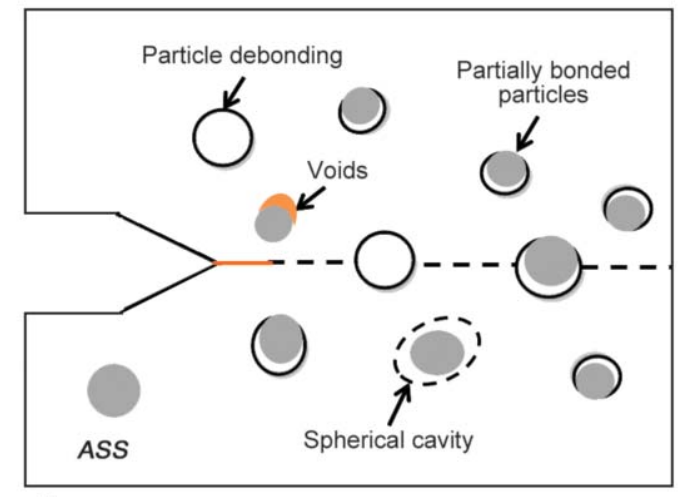

b)

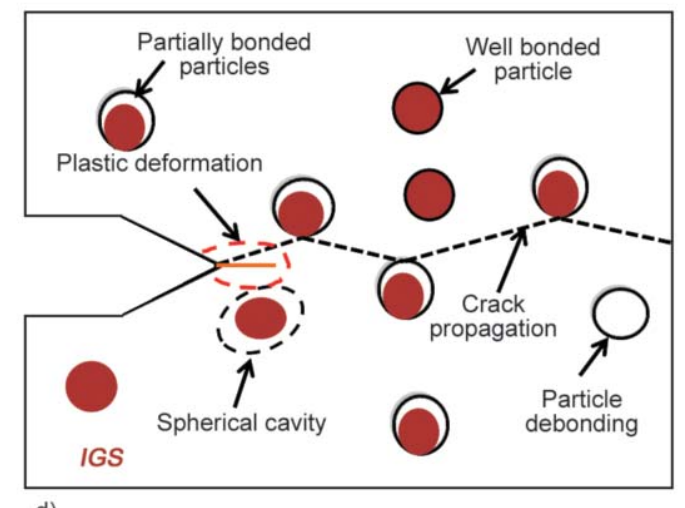

d)

Figure 15. Schematic representation for toughening mechanism involved in the mode-I fracture of epoxy nanocomposites (a) $N E$ (b) ASEC (c) GSEC and (d) ISEC.

GPTMS and imidazole moiety on the surface of ASS was verified using the spectroscopy analysis. The DSC cure study demonstrates that the composite has a good quality of cure at $0.5 \mathrm{wt} \%$ addition of $G G S$ in the epoxy system. The epoxy modified with $G G S$ significantly improved the tensile performance of the nanocomposites. For GSEC, the maximum enhancement of $\sim 58$ and $\sim 21 \%$ in tensile strength and modulus were found with respect to $N E$. The fracture analysis revealed well dispersed $G G S$ in the epoxy system that generated a better interface with the matrix, which absorbed more energy prior to the failure. The tensile fracture surface demonstrated the various failure mechanisms such as particle/matrix interface debonding, particle pullout, and plastic void growth within the matrix. A brittle failure was observed for neat epoxy; however, particle pullout and bondingdebonding within the matrix were observed for $A S E C$ and ISEC. A strong particle-matrix adhesion was observed for $G S E C$, where most of the particles are found well bonded in the epoxy matrix. The well bonded $G G S$ forced the crack to propagate around the pole of the particle by creating a fracture toughen zone resulting in the absorption of more energy. The better interfacial adhesion between $G G S$ and the matrix might have modulated the interfacial stiffness by creating a fracture toughen zone that significantly enhances the fracture toughness by $\sim 97 \%$ for $G S E C$ compared to the $N E$. The significant improvement in the mechanical performance of the nanocomposites displayed the true potential of GGS nanoparticle as reinforcement to mitigate the brittle failure of the epoxy matrix.

\section{Acknowledgements}

The authors thank the Department of Science and Technology, India, under Empowerment and Equity Opportunities for Excellence in Science with Grant No. EEQ/2018/001101 and under DST-FIST Program 2014 with Grant No. SR/FST/ETI$373 / 2014$.

\section{References}

[1] Gu H., Ma C., Gu J., Guo J., Yan X., Huang J., Zhang Q., Guo Z.: An overview of multifunctional epoxy nanocomposites. Journal of Materials Chemistry C, 4, 58905906 (2016).

https://doi.org/10.1039/C6TC01210H 
[2] Kausar A., Rafique I., Muhammad B.: Review of applications of polymer/carbon nanotubes and epoxy/CNT composites. Polymer-Plastics Technology and Engineering, 55, 1167-1191 (2016).

https://oi.org/10.1080/03602559.2016.1163588

[3] Selvam A.: Composites applications in railways. Composites Today, 02, 19-23 (2017).

[4] Kurahatti R. V., Surendranathan A. O., Kori S. A., Singh N., Kumar A. V. R., Srivastava S.: Defence applications of polymer nanocomposites. Defence Science Journal, 60, 551-563 (2010).

https://doi.org/10.14429/dsj.60.578

[5] Singh S., Srivastava V. K., Prakash R.: Influences of carbon nanofillers on mechanical performance of epoxy resin polymer. Applied Nanoscience, 5, 305-313 (2015). https://doi.org/10.1007/s13204-014-0319-0

[6] Conradi M., Zork M., Kocijan A., Verpoest I.: Mechanical properties of epoxy composites reinforced with a low volume fraction of nanosilica fillers. Material Chemistry and Physics, 137, 910-915 (2013). https://doi.org/10.1016/j.matchemphys.2012.11.001

[7] Lee D. W., Yoo B. R.: Advanced silica/polymer composites: Materials and applications. Journal of Industrial and Engineering Chemistry, 38, 1-12 (2016).

https://doi.org/10.1016/j.jiec.2016.04.016

[8] Wang K., Chen L., Wu J., Toh M., He C., Yee A. F.: Epoxy nanocomposites with highly exfoliated clay: Mechanical properties and fracture mechanisms. Macromolecules, 38, 788-800 (2005).

https://doi.org/10.1021/ma048465n

[9] Prasad T., Halder S., Goyat M. S., Dhar S. S.: Morphological dissimilarities of $\mathrm{ZnO}$ nanoparticles and its effect on thermo-physical behavior of epoxy composites. Polymer Composites, 39, 135-145 (2018). https://doi.org/10.1002/pc.23914

[10] Halder S., Prasad T., Khan N. I., Goyat M. S., Chauhan R. S.: Superior mechanical properties of poly vinyl alcohol-assisted $\mathrm{ZnO}$ nanoparticle reinforced epoxy composites. Materials Chemistry and Physics, 192, 198 209 (2017).

https://doi.org/10.1016/j.matchemphys.2016.12.055

[11] Halder S., Ahemad S., Das S., Wang J.: Epoxy/glass fiber laminated composites integrated with amino functionalized $\mathrm{ZrO}_{2}$ for advanced structural applications. ACS Applied Materials and Interfaces, 8, 1695-1706 (2016). https://doi.org/10.1021/acsami.5b09149

[12] Wang H., Sun T., Peng C., Wu Z.: Effect of different silane coupling agents on cryogenic properties of silica-reinforced epoxy composites. High Performance Polymers, 30, 24-37 (2018).

https://doi.org/10.1177/0954008316675207

[13] Mahrholz T., Stängle J., Sinapius M.: Quantitation of the reinforcement effect of silica nanoparticles in epoxy resins used in liquid composite moulding processes. Composites Part A: Applied Science and Manufacturing, 40, 235-243 (2009).

https://doi.org/10.1016/j.compositesa.2008.11.008
[14] Kang S., Hong S. I., Choe C. R., Park M., Rim S., Kim J.: Preparation and characterization of epoxy composites filled with functionalized nanosilica particles obtained via sol-gel process. Polymer, 42, 879-887 (2001). https://doi.org/10.1016/S0032-3861(00)00392-X

[15] Yang Z., Peng H., Wang W., Liu T.: Crystallization behavior of poly( $\varepsilon$-caprolactone)/layered double hydroxide nanocomposites. Journal of Applied Polymer Science, 116, 2658-2667 (2010).

https://doi.org/10.1002/app.31787

[16] Wang C., Jiao G-S., Peng L., Zhu B-L., Li K-Z., Wang J-L.: Influences of surface modification of nano-silica by silane coupling agents on the thermal and frictional properties of cyanate ester resin. Results in Physics, 9 , 886-896 (2018).

https://doi.org/10.1016/j.rinp.2018.03.056

[17] Karmouch R., Ross G. G.: Superhydrophobic wind turbine blade surfaces obtained by a simple deposition of silica nanoparticles embedded in epoxy. Applied Surface Science, 257, 665-669 (2010). https://doi.org/10.1016/j.apsusc.2010.07.041

[18] Hu G., Zhang X., Liu L., Ling W., Cui W.: Study on inert solvent for preparation based on GO/EP composite. Pigment and Resin Technology, 48, 178-184 (2019). https://doi.org/10.1108/PRT-06-2018-0051

[19] Rong M. Z., Zhang M. Q., Ruan W. H.: Surface modification of nanoscale fillers for improving properties of polymer nanocomposites: A review. Materials Science and Technology, 22, 787-796 (2006).

https://doi.org/10.1179/174328406X101247

[20] Rahman I. A., Padavettan V.: Synthesis of silica nanoparticles by sol-gel: Size-dependent properties, surface modification, and applications in silica-polymer nanocomposites - Journal of Nanomaterials, 2012, 132424/1132424/15 (2012).

https://doi.org/10.1155/2012/132424

[21] Kim H. J., Jung D. H., Jung I. H., Cifuentes J. I., Rhee K. Y., Hui D.: Enhancement of mechanical properties of aluminium/epoxy composites with silane functionalization of aluminium powder. Composites Part B: Engineering, 43, 1743-1748 (2012).

https://doi.org/10.1016/j.compositesb.2011.12.010

[22] Han W., Yu Y., Fang L., Johnston M. R., Qiao S. Z., Tang Y.: Functionalised silica/epoxy nanocomposites with enhanced fracture toughness for large-scale applications. Journal of Composite Materials, 49, 14391447 (2015). https://doi.org/10.1177/0021998314534867

[23] Zhang C., Mi X., Tian J., Zhang J., Xu T.: Supported ionic liquid silica as curing agent for epoxy composites with improved mechanical and thermal properties. Polymers, 9, 478/1-478/11 (2017). https://doi.org/10.3390/polym9100478

[24] Hench L. L., West J. K.: The sol-gel process. Chemical Reviews, 90, 33-72 (1990). https://doi.org/10.1021/cr00099a003 
[25] Chen S., Osaka A., Hayakawa S., Tsuru K., Fujii E., Kawabata K.: Novel one-pot sol-gel preparation of amino-functionalized silica nanoparticles. Chemistry Letters, 37, 1170/1-1170/2 (2008). https://doi.org/10.1246/c1.2008.1170

[26] Serantoni da Silveira T. F., Fernandes D. S., Barbosa P. F. P., do Carmo D. R.: Preparation and use of a grafted silica with imidazole groups for cadmium sorption and subsequent voltammetric detection of ascorbic acid. Silicon, 10, 635-643 (2018).

https://doi.org/10.1007/s12633-016-9506-9

[27] Halder S., Goyat M. S., Ghosh P. K.: Influence of ultrasonic dual mode mixing on the morphology, molecular structure and thermo-physical properties of a $\mathrm{SiO}_{2}-$ epoxy nanocomposite adhesive. Journal of Adhesion Science and Technology, 29, 2590-2604 (2015). https://doi.org/10.1080/01694243.2015.1075858

[28] Lee H-H., Lee C-J., Asaoka K.: Correlation in the mechanical properties of acrylic denture base resins. Dental Materials Journal, 31, 157-164 (2012). https://doi.org/10.4012/dmj.2011-205

[29] Karimi A., Navidbakhsh M., Haghi A. M.: An experimental study on the structural and mechanical properties of polyvinyl alcohol sponge using different stressstrain definitions. Advances in Polymer Technology, 33, 21441/1-21441/6 (2014).

https://doi.org/10.1002/adv.21441

[30] Han Y., Lu Z., Teng Z., Liang J., Guo Z., Wang D., Han M-Y., Yang W.: Unraveling the growth mechanism of silica particles in the Stöber method: In situ seeded growth mode. Langmuir, 33, 5879-5890 (2017).

https://doi.org/10.1021/acs.langmuir.7b01140

[31] Kravchenko A., Kolobova E., Kartsova L.: Multifunction covalent coatings for separation of amino acids, biogenic amines, steroid hormones, and ketoprofen enantiomers by capillary electrophoresis and capillary electrochromatography. Separation Science Plus, 3, 102 111 (2020). https://doi.org/10.1002/sscp.201900098

[32] Vollath D., Fischer F. D., Holec D.: Surface energy of nanoparticles - Influence of particle size and structure. Beilstein Journal of Nanotechnology, 9, 2265-2276 (2018). https://doi.org/10.3762/bjnano.9.211

[33] Prasad T., Halder S., Dhar S. S.: Imidazole-supported silica one-pot processed nanoparticles to enhance toughness of epoxy based nanocomposites. Materials Chemistry and Physics, 231, 75-86 (2019). https://doi.org/10.1016/j.matchemphys.2019.04.002

[34] He W., Wu D., Li J., Zhang K., Xiang Y., Long L., Qin S., Yu J., Zhang Q.: Surface modification of colloidal silica nanoparticles: Controlling the size and grafting process. Bulletin of the Korean Chemical Society, 34, 2747-2752 (2013).

https://doi.org/10.5012/bkcs.2013.34.9.2747
[35] Mori T., Okada Y., Kamiya H.: Effect of surface modification of silica particles on interaction forces and dispersibility in suspension. Advance Powder Technology, 27, 830-838 (2015). https://doi.org/10.1016/j.apt.2015.10.020

[36] Chen J., Liu M., Chen C., Gong H., Gao C.: Synthesis and characterization of silica nanoparticles with welldefined thermoresponsive PNIPAM via a combination of RAFT and click chemistry. Applied Materials and Interface, 3, 3215-3223 (2011).

https://doi.org/10.1021/am2007189

[37] Nhavene E. P. F., da Silva W. M., Trivelato Junior R. R., Gastelois P. L., Venâncio T., Nascimento R., Batista R. J. C., Machado C. R., de Almeida Macedo W. A., de Sousa E. M. B.: Chitosan grafted into mesoporous silica nanoparticles as benznidazol carrier for Chagas diseases treatment. Microporous and Mesoporous Materials, 272, 265-275 (2018).

https://doi.org/10.1016/j.micromeso.2018.06.035

[38] Song X., Li D., Li R., Chen Y., Huang L., Wei M., Pan X., Zhou H., Tang H.: Ionic liquid modified inorganic nanoparticles for gaseous phenol adsorption. Journal of Wuhan University of Technology, Materials Science Edition, 34, 787-790 (2019). https://doi.org/10.1007/s11595-019-2118-7

[39] Jouyandeh M., Jajani O. M., Navarchian A. H., Shabanian M., Vahabi H., Saeb M. R.: Bushy-surface hybrid nanoparticles for developing epoxy superadhesives. Applied Surface Science, 479, 1148-1160 (2019). https://doi.org/10.1016/j.apsusc.2019.01.283

[40] Jouyandeh M., Paran S. M. R., Shabanian M., Ghiyasi S., Vahabi H., Badawi M., Formela K., Puglia D., Saeb M. R.: Curing behavior of epoxy $/ \mathrm{Fe}_{3} \mathrm{O}_{4}$ nanocomposites: A comparison between the effects of bare $\mathrm{Fe}_{3} \mathrm{O}_{4}$, $\mathrm{Fe}_{3} \mathrm{O}_{4} / \mathrm{SiO}_{2} /$ chitosan and $\mathrm{Fe}_{3} \mathrm{O}_{4} / \mathrm{SiO}_{2} /$ chitosan/imide/ phenylalanine-modified nanofillers. Progress in Organic Coating, 123, 10-19 (2018). https://doi.org/10.1016/j.porgcoat.2018.06.006

[41] Jouyandeh M., Paran S. M. R., Jannesari A., Saeb M. R.: 'Cure index' for thermoset composites. Progress in Organic Coating, 127, 429-434 (2019).

https://doi.org/10.1016/j.porgcoat.2018.11.025

[42] Ratna D.: Modification of epoxy resins for improvement of adhesion: A critical review. Journal of Adhesion Science and Technology, 17, 1655-1668 (2003). https://doi.org/10.1163/156856103322396721

[43] Yin C., Zhang Q., Gu J., Zhao Z., Zheng J., Gong G., Liang T., Zhang H.: Cure characteristics and mechanical properties of vinyltriethoxysilane grafted styrenebutadiene rubber/silica blends. Polymer-Plastics Technology and Engineering, 51, 1218-1222 (2012). https://doi.org/10.1080/03602559.2012.696765

[44] Roy S., Petrova R. S., Mitra S.: Effect of carbon nanotube (CNT) functionalization in epoxy-CNT composites. Nanotechnology Reviews, 7, 475-485 (2018). https://doi.org/10.1515/ntrev-2018-0068 
[45] Sae-oui P., Sirisinha C., Thepsuwan U., Hatthapanit K.: Roles of silane coupling agents on properties of silicafilled polychloroprene. European Polymer Journal, 42, 479-486 (2006).

https://doi.org/10.1016/j.eurpolymj.2005.09.003

[46] Afzal A., Siddiqi H. M., Saeed S., Ahmad Z.: The influence of epoxy functionalized silica nanoparticles on stress dispersion and crack resistance in epoxy-based hybrids. Material Express, 1, 299-306 (2011). https://doi.org/10.1166/mex.2011.1042

[47] Jux M., Fankhänel J., Daum B., Mahrholz T., Sinapius M., Rolfes R.: Mechanical properties of epoxy/boehmite nanocomposites in dependency of mass fraction and surface modification - An experimental and numerical approach. Polymer, 141, 34-45 (2018).

https://doi.org/10.1016/j.polymer.2018.02.059

[48] Lauke B.: On the effect of particle size on fracture toughness of polymer composites. Composites Science and Technology, 68, 3365-3372 (2008).

https://doi.org/10.1016/j.compscitech.2008.09.011

[49] Goyat M. S., Rana S., Halder S., Ghosh P. K.: Facile fabrication of epoxy- $\mathrm{TiO}_{2}$ nanocomposites: A critical analysis of $\mathrm{TiO}_{2}$ impact on mechanical properties and toughening mechanisms. Ultrasonics Sonochemistry, 40, 861-873 (2018).

https://doi.org/10.1016/j.ultsonch.2017.07.040

[50] Hsieh T. H., Kinloch A. J., Masania K., Taylor A. C., Sprenger S.: The mechanisms and mechanics of the toughening of epoxy polymers modified with silica nanoparticles. Polymer, 51, 6284-6294 (2010).

https://doi.org/10.1016/j.polymer.2010.10.048

[51] Das S., Halder S., Wang J., Goyat M. S., Kumar A. A., Fang Y.: Amending the thermo-mechanical response and mechanical properties of epoxy composites with silanized chopped carbon fibers. Composites Part A: Applied Science and Manufacturing, 102, 347-356 (2017).

https://doi.org/10.1016/j.compositesa.2017.07.026
[52] Dai J., Peng C., Wang F., Zhang G., Huang Z.: Effects of functionalized graphene nanoplatelets on the morphology and properties of phenolic resins. Journal of Nanomaterials, 2016, 3485167/1-3485167/7 (2016). https://doi.org/10.1155/2016/3485167

[53] Feng Y., Chen H., Dong X., Qu J., He H., Xu B., Zhang Y.: Polyvinyl alcohol-modified Pithecellobium clypearia benith herbal residue fiber/polypropylene composites. Polymer Composites, 37, 915-924 (2016). https://doi.org/10.1002/pc. 23250

[54] Chandrasekaran S., Sato N., Tölle F., Mülhaupt R., Fiedler B., Schulte K.: Fracture toughness and failure mechanism of graphene based epoxy composites. Composites Science and Technology, 97, 90-99 (2014). https://doi.org/10.1016/j.compscitech.2014.03.014

[55] Hedrick J. L., Yilgor I., Jurek M., Hedrick J. C., Wilkes G. L., Mcgrath J. E.: Chemical modification of matrix resin networks with engineering thermoplastics: 1 . Synthesis, morphology, physical behaviour and toughening mechanisms of poly(arylene ether sulphone) modified epoxy networks. Polymer, 32, 2020-2032 (1991). https://doi.org/10.1016/0032-3861(91)90168-I

[56] Naebe M., Abolhasani M. M., Khayyam H., Amini A., Fox B.: Crack damage in polymers and composites: A review. Polymer Reviews, 56, 31-69 (2016). https://doi.org/10.1080/15583724.2015.1078352

[57] Beaumont P. W. R., Young R. J.: Failure of brittle polymers by slow crack growth. Journal of Materials Science, 10, 1334-1342(1975). https://doi.org/10.1007/BF00540823 